\title{
Point-free characterisation of Bishop compact metric spaces
}

\author{
TATSUJI KAWAI
}

\begin{abstract}
We give a point-free characterisation of Bishop compact metric spaces in terms of formal topology. We show that the notion of overt compact enumerably completely regular formal topology is a point-free counterpart of that of Bishop compact metric space. Specifically, a formal topology is isomorphic to an overt compact enumerably completely regular formal topology if and only if it is isomorphic to the image of a compact metric space under the localic completion of metric spaces into formal topologies. The result is obtained in Bishop constructive mathematics with the axiom of Dependent Choice.
\end{abstract}

2010 Mathematics Subject Classification 03F60 (primary); 06D22, 54E45 (secondary)

Keywords: Formal topologies, Compact metric spaces, Localic completion, Bishop constructive mathematics

\section{Introduction}

Bishop [2] developed a large body of analysis constructively, but he did not develop general topology beyond the theory of metric spaces. He found it difficult to find a useful topological notion of compactness which is compatible with the corresponding metric notion defined by completeness and totally boundedness. If the classical notion of compactness by open cover were adopted, there would be no nontrivial examples of compact spaces constructively. In fact, the main examples of compact metric spaces, the unit interval and Cantor space, cannot be proved to be compact in the sense of open cover without recourse to Fan theorem, which is constructively unacceptable.

The study of general topology in a constructive setting was initiated by Sambin [15], when he introduced the notion of formal topology, a point-free approach to general topology based on the impredicative theory of locale (Johnstone [11]). Formal topology has been successful in constructivising many results of classical topology; however, the connection between Bishop's metric space and formal topology has been somewhat neglected until recently. In particular, the notion of compactness in formal topology, 
which is defined by open cover, seems to conflict with the compactness of Bishop metric space defined by completeness and totally boundedness.

Palmgren [14], in his pioneering work in connecting Bishop metric spaces and formal topologies, extended the localic completion of generalised metric spaces due to Vickers [17] into a full and faithful functor from the category of Bishop locally compact metric spaces into that of locally compact formal topologies. The functor can be restricted to the full subcategory of compact metric spaces and that of compact formal topologies. Thus, the two seemingly conflicting notions of compactness are actually compatible. Later, Spitters [16] and Coquand et al. [5] found a connection between the compact subspaces of a Bishop locally compact metric space and the compact overt subtopologies of its localic completion.

In this paper, building on these previous works, we characterise the image of compact metric spaces under the Palmgren's embedding (ie a full and faithful functor) in terms of formal topology. Our result gives a purely point-free characterisation of Bishop compact metric spaces, and it allows us to prove results about Bishop compact metric spaces in a point-free way.

In our subsequent paper [12], we extend our point-free characterisation of compact metric spaces to Bishop locally compact metric spaces.

The content of this paper is as follows. Section 2 and Section 3 are preliminaries. In Section 2, we give background on formal topology. In Section 3, we review the notion of localic completion by Vickers and Palmgren's functorial embedding of the category of locally compact metric spaces into that of formal topologies. In Section 4, we show that the localic completion induces a bijection between the compact subspaces of a locally compact metric space and the compact overt subtopologies of its localic completion (Theorem 4.16). As a corollary, we obtain a preliminary characterisation of formal topologies that are isomorphic to the image of some compact metric space under the localic completion (Corollary 4.17). In Section 5, we show that the embedding preserves countable products of inhabited compact metric spaces (Theorem 5.4). Finally, in Section 6, we give a point-free characterisation of Bishop compact metric spaces (Theorem 6.10).

We work constructively in the sense of Bishop [2], including the axiom of Dependent Choice. However, the intended formal system underlying our work is Aczel's constructive set theory CZF [1] extended with the Regular Extension Axiom (REA) and Dependent Choice. Hence, when we say that an object $A$ is a set, it means that $A$ forms a set in CZF, and when we say that $A$ is a class, it means that $A$ may not be a set but its members can be specified by a formula of CZF. 
The axiom REA is needed to define the notion of inductively generated formal topology (Section 2.3). However, the results on locally compact metric spaces, including the main result in Section 6, do not require inductive generation of formal topologies, and hence do not require REA (see Remark 3.7).

We recall that Dependent Choice is the following principle:

DC Given a set $A$, a total (set) relation $R \subseteq A \times A$ and $a_{0} \in A$, there exists a function $f: \mathbb{N} \rightarrow A$ such that $f(0)=a_{0}$ and $f(n) R f(n+1)$ for all $n \in \mathbb{N}$.

\section{Preliminaries}

First, we fix some notations. Given a set $S, \operatorname{Pow}(S)$ denotes the class of subsets of $S$. Constructively, $\operatorname{Pow}(S)$ is not a set unless $S=\emptyset$. Fin $(S)$ denotes the set of finitely enumerable subsets of $S$, where a set $A$ is finitely enumerable if there exists a surjection $f:\{0, \ldots, n-1\} \rightarrow A$ for some $n \in \mathbb{N}$. For subsets $U, V \subseteq S$, we define

$$
U \curlyvee V \stackrel{\text { def }}{\Longleftrightarrow}(\exists a \in S) a \in U \cap V .
$$

The set theoretic complement of a subset $U \subseteq S$ is denoted by $\neg U$.

If $r$ is a relation between sets $X$ and $S$, we write $r^{-}$for the inverse relation of $r$. The direct image of a subset $D \subseteq X$ under $r$ is defined by $r D=\{a \in S \mid(\exists x \in D) \times r a\}$. In particular, if $U$ is a subset of $S$, its inverse image under $r$ is $r^{-} U$. For a singleton $\{x\} \subseteq X$, we sometimes write $r x$ for $r\{x\}$. Furthermore, we introduce the following notation associated with a relation $r$ :

$$
r^{*} U \stackrel{\text { def }}{=}\{x \in X \mid r\{x\} \subseteq U\} .
$$

If $r^{-}$is the inverse relation of $r$, we write $r^{-*}$ for $\left(r^{-}\right)^{*}$.

\subsection{Formal topologies}

We recall the relevant facts about formal topology used in this paper. Our presentation is based on the work by Fox [10], and it is compatible with that of Palmgren [14].

Definition 2.1 A formal topology is a triple $\mathcal{S}=(S, \triangleleft, \leq)$ where $(S, \leq)$ is a preordered set and $\triangleleft$ is a relation between $S$ and $\operatorname{Pow}(S)$ such that

$$
\mathcal{A} U \stackrel{\text { def }}{=}\{a \in S \mid a \triangleleft U\}
$$

is a set for each $U \subseteq S$, and satisfies 
(Ref) $U \triangleleft U$,

(Tra) $a \triangleleft U \& U \triangleleft V \Longrightarrow a \triangleleft V$,

(Loc) $a \triangleleft U \& a \triangleleft V \Longrightarrow a \triangleleft U \downarrow V$,

(Ext) $a \leq b \Longrightarrow a \triangleleft b$

for all $a, b \in S$ and $U, V \subseteq S$, where

$$
\begin{aligned}
& U \triangleleft V \stackrel{\text { def }}{\Longleftrightarrow}(\forall a \in U) a \triangleleft V, \\
& U \downarrow V \stackrel{\text { def }}{=}\{c \in S \mid(\exists a \in U)(\exists b \in V) c \leq a \& c \leq b\} .
\end{aligned}
$$

We write $a \downarrow U$ for $\{a\} \downarrow U$, and $U \triangleleft a$ for $U \triangleleft\{a\}$. The underlying set $S$ is called the base, and the relation $\triangleleft$ is call the cover on $S$.

It is well known that the class $\operatorname{Sat}(\mathcal{S})=\{\mathcal{A} U \mid U \in \operatorname{Pow}(S)\}$ forms a frame; it can be identified with $\operatorname{Pow}(S)$ together with the equality

$$
U=\mathcal{S} V \stackrel{\text { def }}{\Longleftrightarrow} \mathcal{A} U=\mathcal{A} V .
$$

Notation 1 Letters $\mathcal{S}, \mathcal{S}^{\prime}, \ldots$ denote formal topologies, whose underlying bases, covers, and preorders are denoted by $S, S^{\prime}, \ldots, \triangleleft, \triangleleft^{\prime}, \ldots$, and $\leq, \leq^{\prime}, \ldots$, respectively.

Definition 2.2 Let $\mathcal{S}$ and $\mathcal{S}^{\prime}$ be formal topologies. A relation $r \subseteq S \times S^{\prime}$ is a formal topology map from $\mathcal{S}$ to $\mathcal{S}^{\prime}$ if

(FTM1) $S \triangleleft r^{-} S^{\prime}$,

(FTM2) $r^{-}\{a\} \downarrow r^{-}\{b\} \triangleleft r^{-}\left(a \downarrow^{\prime} b\right)$,

(FTM3) $a \triangleleft^{\prime} U \Longrightarrow r^{-} a \triangleleft r^{-} U$

for all $a, b \in S^{\prime}$ and $U \subseteq S^{\prime}$. The class of formal topology maps from $\mathcal{S}$ to $\mathcal{S}^{\prime}$ is equipped with the equality

$$
r=s \stackrel{\text { def }}{\Longleftrightarrow}\left(\forall a \in S^{\prime}\right) r^{-}\{a\}=s s^{-}\{a\} .
$$

A point of a formal topology $\mathcal{S}$ is a formal topology map from the terminal formal topology $\mathbf{1}=(\{*\}, \in,=)$ to $\mathcal{S}$. An equivalent description is the following.

Definition 2.3 A subset $\alpha \subseteq S$ is a formal point of $\mathcal{S}$ if

(P1) $S \oint \alpha$,

(P2) $a, b \in \alpha \Longrightarrow \alpha \gamma(a \downarrow b)$,

(P3) $a \in \alpha \& a \triangleleft U \Longrightarrow \alpha \curlyvee U$ 
for all $a, b \in S$ and $U \subseteq S$. The class of formal points of $\mathcal{S}$ is denoted by $\operatorname{Pt}(\mathcal{S}){ }^{1}$

Formal topologies and formal topology maps form a category, which we denote by FTop.

\subsection{Overt formal topologies}

We first recall the notion of formal closed subset, which can be seen as a point-free analogue of a closed subset (cf Theorem 4.3). In locale theory, formal closed subsets are known as the points of the lower powerlocales; see Vickers [19] for details.

Definition 2.4 A subset $V \subseteq S$ of a formal topology $\mathcal{S}$ is formal closed if

$$
a \in V \& a \triangleleft U \Longrightarrow V \curlyvee U
$$

for all $a \in S$ and $U \subseteq S$. The class of formal closed subsets of $\mathcal{S}$ is denoted by $\operatorname{Red}(\mathcal{S})$.

Note that every formal point of $\mathcal{S}$ is formal closed, ie $\operatorname{Pt}(\mathcal{S}) \subseteq \operatorname{Red}(\mathcal{S})$.

Definition 2.5 Let $\mathcal{S}$ be a formal topology. A positivity predicate on $\mathcal{S}$ is a formal closed subset Pos $\subseteq S$ that satisfies

$$
a \triangleleft\{x \in S \mid x=a \& \operatorname{Pos}(a)\}
$$

for all $a \in S$, where we write $\operatorname{Pos}(a)$ for $a \in \operatorname{Pos}$. Note that

$$
\{x \in S \mid x=a \& \operatorname{Pos}(a)\}=\{a\} \cap \operatorname{Pos} .
$$

A formal topology is overt if it is equipped with a (necessarily unique) positivity predicate. $^{2}$

\subsection{Inductively generated formal topologies}

An axiom-set on a set $S$ is a pair $(I, C)$, where $(I(a))_{a \in S}$ is a family of sets indexed by $S$, and $C$ is a family $(C(a, i))_{a \in S, i \in I(a)}$ of subsets of $S$ indexed by $\sum_{a \in S} I(a)$.

\footnotetext{
${ }^{1}$ In CZF, the class of formal points of $\mathcal{S}$ is not a set in general.

${ }^{2} \mathrm{An}$ overt formal topology is called an open formal topology in Fox [10]. In this paper, we follow Spitters [16] for terminology.
}

Journal of Logic \& Analysis 9:5 (2017) 
Theorem 2.6 (Coquand et al. [6]) Let $(S, \leq)$ be a preordered set, and let $(I, C)$ be an axiom set on $S$. Then, there exists a cover $\triangleleft_{I, C}$ inductively generated by the following rules:

$$
\begin{aligned}
& \frac{a \in U}{a \triangleleft_{I, C} U} \text { (reflexivity), } \frac{a \leq b \quad b \triangleleft_{I, C} U}{a \triangleleft_{I, C} U}(\leq \text {-left), } \\
& \frac{a \leq b \quad i \in I(b) \quad a \downarrow C(b, i) \triangleleft_{I, C} U}{a \triangleleft_{I, C} U}(\leq \text {-infinity). }
\end{aligned}
$$

The relation $\triangleleft_{I, C}$ is the least cover on $S$ which satisfies ( $\leq-$ left) and $a \triangleleft_{I, C} C(a, i)$ for each $a \in S$ and $i \in I(a)$, and $\triangleleft_{I, C}$ is called the cover inductively generated by $(I, C)$.

Moreover, suppose that a subset Pos $\subseteq S$ is given which satisfies the conditions

(Spl1) $a \leq b \& \operatorname{Pos}(a) \Longrightarrow \operatorname{Pos}(b)$,

(Spl2) $a \leq b \& \operatorname{Pos}(a) \Longrightarrow \operatorname{Pos} \gamma(a \downarrow C(b, i))$

for each $a, b \in S$ and $i \in I(b)$. Let $\triangleleft_{I^{\prime}, C^{\prime}}$ be the cover inductively generated by the axiom-set $\left(I^{\prime}, C^{\prime}\right)$ obtained from $(I, C)$ by adding one axiom

$$
a \triangleleft_{I^{\prime}, C^{\prime}} \operatorname{Pos} \cap\{a\}
$$

for each $a \in S$. Then, the formal topology $\mathcal{S}=\left(S, \triangleleft_{I^{\prime}, C^{\prime}}, \leq\right)$ is overt with the positivity Pos, and the cover $\triangleleft_{I^{\prime}, C^{\prime}}$ is the least cover on $S$ which satisfies ( $\leq$-left) and $a \triangleleft_{I^{\prime}, C^{\prime}} C(a, i)$ for each $a \in S$ and $i \in I(a)$, and for which Pos is a positivity predicate on $\mathcal{S}$.

A formal topology $\mathcal{S}=(S, \triangleleft, \leq)$ is inductively generated if there exists an axiom-set $(I, C)$ on $S$ which generates the cover $\triangleleft$ as in Theorem 2.6.

Remark 2.7 If $r: \mathcal{S} \rightarrow \mathcal{S}^{\prime}$ is a formal topology map and $\mathcal{S}^{\prime}$ is inductively generated by an axiom-set $(I, C)$ on $S^{\prime}$, then the condition (FTM3) is equivalent to the following conditions under the condition (FTM2):

(FTM3a) $a \leq^{\prime} b \Longrightarrow r^{-} a \triangleleft r^{-} b$,

(FTM3b) $\quad r^{-} a \triangleleft r^{-} C(a, i)$

for all $a, b \in S^{\prime}$ and $i \in I(a)$.

If $\mathcal{S}$ is inductively generated by an axiom-set $(I, C)$ on $S$, then a subset $V \subseteq S$ is formal closed if and only if $V$ satisfies the conditions (Spl1) and (Spl2) in Theorem 2.6. 
Example 2.8 (The formal reals; Negri and Soravia [13], Coquand et al. [6]) Let $\mathbb{Q}$ be the set of rationals. Define a preorder $\left(S_{\mathcal{R}}, \leq_{\mathcal{R}}\right)$ by

$$
\begin{aligned}
& S_{\mathcal{R}} \stackrel{\text { def }}{=}\{(p, q) \in \mathbb{Q} \times \mathbb{Q} \mid p<q\}, \\
& (p, q) \leq_{\mathcal{R}}(r, s) \stackrel{\text { def }}{\Longleftrightarrow} r \leq p \& q \leq s
\end{aligned}
$$

for all $(p, q),(r, s) \in S_{\mathcal{R}}$. The formal reals $\mathcal{R}=\left(S_{\mathcal{R}}, \triangleleft_{\mathcal{R}}, \leq_{\mathcal{R}}\right)$ is inductively generated by an axiom-set on $S_{\mathcal{R}}$ consisting of axioms

(R1) $(p, q) \triangleleft_{\mathcal{R}}\left\{(r, s) \in S_{\mathcal{R}} \mid p<r<s<q\right\}$,

(R2) $(p, q) \triangleleft_{\mathcal{R}}\{(p, s),(r, q)\}$ for each $p<r<s<q$.

It is well known that the formal points of $\mathcal{R}$ correspond to the Dedekind cuts.

\subsection{Closed and weakly closed subtopologies}

Definition 2.9 A subtopology of a formal topology $\mathcal{S}$ is a formal topology $\mathcal{S}^{\prime}=$ $\left(S, \triangleleft^{\prime}, \leq\right)$ where $\triangleleft^{\prime}$ is a cover on $S$ and $(S, \leq)$ is the underlying preorder of $\mathcal{S}$ such that $\mathcal{A} U \subseteq \mathcal{A}^{\prime} U$ for all $U \subseteq S$. If $\mathcal{S}^{\prime}$ is a subtopology of $S$, we write $\mathcal{S}^{\prime} \sqsubseteq \mathcal{S}$.

Let $r: \mathcal{S}^{\prime} \rightarrow \mathcal{S}$ be a formal topology map. The image of $\mathcal{S}^{\prime}$ under $r$ is a subtopology $\mathcal{S}_{r}=\left(S, \triangleleft_{r}, \leq\right)$ of $\mathcal{S}$ where

$$
a \triangleleft_{r} U \stackrel{\text { def }}{\Longleftrightarrow} r^{-} a \triangleleft^{\prime} r^{-} U .
$$

A formal topology map $r: \mathcal{S}^{\prime} \rightarrow \mathcal{S}$ is an embedding if

$$
a \triangleleft^{\prime} r^{-} r^{-*} \mathcal{A}^{\prime}\{a\}
$$

for all $a \in S^{\prime}$. It can be shown that the domain of an embedding is isomorphic to its image; see Fox [10, Section 3.5].

The following is well known. We omit a straightforward proof.

Lemma 2.10 Let $\mathcal{S}$ be an overt formal topology with a positivity Pos, and let $r: \mathcal{S} \rightarrow \mathcal{S}^{\prime}$ be a formal topology map. Then, the image $\mathcal{S}_{r}$ is overt with the positivity

$$
r \text { Pos }=\left\{a \in S^{\prime} \mid(\exists b \in \text { Pos }) b r a\right\} .
$$

Definition 2.11 Each subset $V \subseteq S$ of a formal topology $\mathcal{S}$ determines a subtopology $\mathcal{S}^{\mathcal{S}-V}=\left(S, \triangleleft^{\mathcal{S}-V}, \leq\right)$ whose cover is defined by

$$
a \triangleleft^{\mathcal{S}-V} U \stackrel{\text { def }}{\Longleftrightarrow} a \triangleleft V \cup U .
$$


A subtopology of $\mathcal{S}$ of the form $\mathcal{S}^{\mathcal{S}-V}$ is called the closed subtopology (determined by $V$ ). The closure of a subtopology $\mathcal{S}^{\prime}$ of $\mathcal{S}$ is the closed subtopology $\mathcal{S}^{\mathcal{S}-Z}$ determined by $Z \stackrel{\text { def }}{=}\left\{a \in S \mid a \triangleleft^{\prime} \emptyset\right\}$.

The closed subtopology $\mathcal{S}^{\mathcal{S}-V}$ is the largest subtopology $\mathcal{S}^{\prime}$ of $\mathcal{S}$ which satisfies $V \triangleleft^{\prime} \emptyset$. The closure of a subtopology $\mathcal{S}^{\prime}$ is the smallest closed subtopology of $\mathcal{S}$ containing $\mathcal{S}^{\prime}$. Note that $V \triangleleft W$ if and only if $\mathcal{S}^{\mathcal{S}-W} \sqsubseteq \mathcal{S}^{\mathcal{S}-V}$.

Example 2.12 Let $\mathcal{R}$ be the formal reals (see Example 2.8). The formal unit interval $\mathcal{I}[0,1]$ is a closed subtopology of $\mathcal{R}$ determined by the subset $(-\infty, 0) \cup(1, \infty)$ where

$$
\begin{aligned}
(-\infty, 0) & \stackrel{\text { def }}{=}\left\{(p, q) \in S_{\mathcal{R}} \mid q=0\right\}, \\
(1, \infty) & \stackrel{\text { def }}{=}\left\{(p, q) \in S_{\mathcal{R}} \mid p=1\right\} .
\end{aligned}
$$

Lemma 2.13 Let $\mathcal{S}^{\prime}$ be an overt subtopology of $\mathcal{S}$ with a positivity Pos. Then, the closure of $\mathcal{S}^{\prime}$ in $\mathcal{S}$ is the closed subtopology $\mathcal{S}^{\mathcal{S}-\neg \text { Pos }}$.

Proof Put $Z=\left\{a \in S \mid a \triangleleft^{\prime} \emptyset\right\}$. It suffices to show that $\neg$ Pos $=Z$. Since Pos is a positivity of $\mathcal{S}^{\prime}$, we have $\neg \operatorname{Pos} \triangleleft^{\prime} \emptyset$, and thus $\neg$ Pos $\subseteq Z$. Conversely, if $a \triangleleft^{\prime} \emptyset$ and $a \in$ Pos, then we have $\operatorname{Pos} \gamma \emptyset$, a contradiction. Hence $Z \subseteq \neg$ Pos.

In particular, if $\mathcal{S}^{\prime}$ is an overt closed subtopology of $\mathcal{S}$ with a positivity Pos, then we have $\mathcal{S}^{\prime}=\mathcal{S}^{\mathcal{S}-\neg \text { Pos }}$. In this case, we have

$$
a \triangleleft \neg \operatorname{Pos} \cup(\operatorname{Pos} \cap\{a\}) .
$$

Conversely, formal closed subsets of $\mathcal{S}$ that satisfy the property (2-1) characterise overt closed subtopologies of $\mathcal{S}$. This observation is due to one of the anonymous referees of this paper.

Proposition 2.14 Let $\mathcal{S}$ be a formal topology. There exists an order preserving bijection between overt closed subtopologies of $\mathcal{S}$ and formal closed subsets of $\mathcal{S}$ with the property (2-1).

Proof We have seen that the positivity of an overt closed subtopology satisfies (2-1).

Conversely, let Pos be a formal closed subset of $\mathcal{S}$ with the property (2-1). Then, it is easy to see that Pos is a formal closed subset of the closed subtopology $\mathcal{S}^{\mathcal{S}-\neg \text { Pos }}$. Then, the property (2-1) says that Pos is the positivity of $\mathcal{S}^{\mathcal{S}-\neg \text { Pos }}$. 
Let Pos, Pos ${ }^{\prime}$ be formal closed subsets of $\mathcal{S}$ with the property (2-1). Clearly, Pos $\subseteq$ Pos $^{\prime}$

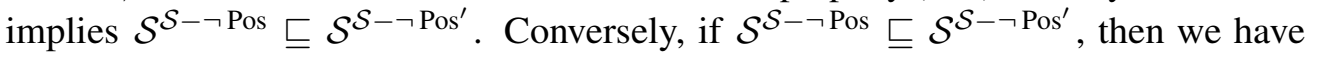
$\neg \operatorname{Pos}^{\prime} \triangleleft \neg$ Pos. Let $a \in$ Pos. Since $a \triangleleft \neg \operatorname{Pos}^{\prime} \cup\left(\operatorname{Pos}^{\prime} \cap\{a\}\right)$ and Pos is formal closed, we have either $\operatorname{Pos} \gamma \neg \neg \operatorname{Pos}^{\prime}$ or $\operatorname{Pos}^{\prime}(a)$. In the former case, we obtain $\operatorname{Pos} \gamma \neg \operatorname{Pos}$, a contradiction. Hence, $\operatorname{Pos} \subseteq$ Pos' $^{\prime}$.

Proposition 2.15 Let $\mathcal{S}^{\prime}$ be an overt closed subtopology of $\mathcal{S}$ with a positivity Pos. Then, $\mathcal{S}^{\prime}$ is the largest subtopology of $\mathcal{S}$ with the positivity Pos.

Proof Let $\mathcal{S}^{\prime \prime}$ be an overt subtopology of $\mathcal{S}$ with the positivity Pos. By Lemma 2.13,

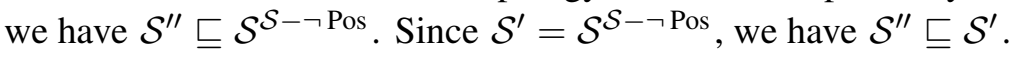

Constructively, there is another notion of closed subtopology, called weakly closed subtopology. We only consider weakly closed subtopology which is also overt; see Vickers [19] and Fox [10] for the treatments in formal topology, and Bunge and Funk [3] for the corresponding notion in locale theory.

Definition 2.16 Let $\mathcal{S}^{\prime}$ be an overt subtopology of $\mathcal{S}$ with a positivity Pos. Then, $\mathcal{S}^{\prime}$ is weakly closed if $\mathcal{S}^{\prime}$ is the largest overt subtopology of $\mathcal{S}$ with the positivity Pos.

Remark 2.17 The notion of overt weakly closed subtopology given in Definition 2.16 is stronger than the one given by Fox [10, Definition 3.5.13], although impredicatively they are equivalent. However, our main examples of overt weakly closed subtopologies, namely overt closed subtopologies, do satisfy the condition of Definition 2.16. Moreover, if a formal topology $\mathcal{S}$ in Definition 2.16 is inductively generated, the two notions coincide.

The following is immediate from Proposition 2.15.

Proposition 2.18 An overt closed subtopology is weakly closed.

Given an inductively generated formal topology $\mathcal{S}$, there exists an order isomorphism between the formal closed subsets of $\mathcal{S}$ and the overt weakly closed subtopologies of $\mathcal{S}$. In fact, by Remark 2.7 and the second half of Theorem 2.6, a formal closed subset $V \subseteq S$ determines an overt inductively generated subtopology $\mathcal{S}$, which we denote by $\mathcal{S}_{V}$. From the axioms of $\mathcal{S}_{V}$, it is easy to see that $\mathcal{S}_{V}$ is the largest subtopology of $\mathcal{S}$ with the positivity $V$. Hence $\mathcal{S}_{V}$ is a weakly closed subtopology. It is straightforward to show that this correspondence is an order isomorphism. Moreover, we have

$$
\operatorname{Pt}\left(\mathcal{S}_{V}\right)=\{\alpha \in \operatorname{Pt}(\mathcal{S}) \mid \alpha \subseteq V\} .
$$

Corollary 2.19 Let $\mathcal{S}$ be an inductively generated formal topology, and let $\mathcal{S}^{\prime} \sqsubseteq \mathcal{S}$ be an overt closed subtopology with a positivity Pos. Then $\mathcal{S}^{\prime}=\mathcal{S}_{\text {Pos }}=\mathcal{S}^{\mathcal{S}-\neg \text { Pos }}$. 


\subsection{Regularity and compactness}

Definition 2.20 Let $\mathcal{S}$ be a formal topology, and let $a, b \in S$. We say that $a$ is well covered by $b$, written $a \lll b$, if $S \triangleleft a^{*} \cup\{b\}$ where $a^{*} \stackrel{\text { def }}{=}\{b \in S \mid b \downarrow a \triangleleft \emptyset\}$.

A formal topology $\mathcal{S}$ is regular if there exists a function wc: $S \rightarrow \operatorname{Pow}(S)$ such that

(1) $(\forall b \in \operatorname{wc}(a)) b \lll a$,

(2) $a \triangleleft \operatorname{wc}(a)$

for each $a \in S$. We often regard wc as a relation $\overline{\mathrm{wC}} \subseteq S \times S$ defined by

$$
\overline{\mathrm{WC}} \stackrel{\text { def }}{=}\{(a, b) \in S \times S \mid a \in \mathrm{wc}(b)\} .
$$

For later use, we extend the relation $\lll$ to the subsets of $S$ by $U \lll V \stackrel{\text { def }}{\Longleftrightarrow} S \triangleleft U^{*} \cup V$ where $U^{*} \stackrel{\text { def }}{=} \bigcap_{a \in U} a^{*}$. We have $U^{\prime} \triangleleft U \lll V \triangleleft V^{\prime} \Longrightarrow U^{\prime} \lll V^{\prime}$. If $r: \mathcal{S} \rightarrow \mathcal{S}^{\prime}$ is a formal topology map and $U \lll^{\prime} V$ in $\mathcal{S}^{\prime}$, then $r^{-} U \lll r^{-} V$ in $\mathcal{S}$.

A formal topology $\mathcal{S}$ is compact if

$$
S \triangleleft U \Longrightarrow\left(\exists U_{0} \in \operatorname{Fin}(U)\right) S \triangleleft U_{0}
$$

for all $U \subseteq S$.

The following are well known in locale theory; see Johnstone [11, Chapter III, Proposition 1.2]. The corresponding results in formal topology were obtained by Curi [9].

\section{Proposition 2.21}

(1) A subtopology of a regular formal topology is regular.

(2) A closed subtopology of a compact formal topology is compact.

(3) A compact subtopology of a regular formal topology is closed.

Definition 2.22 Let $\mathcal{S}$ be a formal topology, and let $a, b \in S$. We say that $a$ is way below $b$, denoted by $a \ll b$, if

$$
b \triangleleft U \Longrightarrow\left(\exists U_{0} \in \operatorname{Fin}(U)\right) a \triangleleft U_{0}
$$

for all $U \subseteq S$. A formal topology $\mathcal{S}$ is locally compact if there exists a function wb: $S \rightarrow \operatorname{Pow}(S)$ such that

(1) $(\forall b \in \mathrm{wb}(a)) b \ll a$,

(2) $a \triangleleft \mathrm{wb}(a)$ 
for each $a \in S$.

In compact regular formal topologies, the two relations $\lll$ and $\ll$ coincides; see Johnstone [11, Chapter VII, Section 3.5]. For the proof in formal topology, see Curi [7, Proposition 2.9].

Proposition 2.23 Every compact regular formal topology is locally compact.

\section{Localic completion of metric spaces}

\subsection{Localic completion}

We recall the representation of complete metric spaces by formal topologies, called localic completion, due to Vickers [17].

Definition 3.1 Let $X=(X, \rho)$ be a metric space, and let $\mathbb{Q}^{>0}$ be the set of positive rationals. Then, a pair $(x, \varepsilon) \in X \times \mathbb{Q}^{>0}$, denoted by $\mathrm{b}(x, \varepsilon)$, is called a formal ball. We write $M_{X}$ for $X \times \mathbb{Q}^{>0}$, the set of formal balls of $X$. Define an order $\leq_{X}$ and a transitive relation $<_{X}$ on $M_{X}$ by

$$
\begin{aligned}
& \mathrm{b}(x, \delta) \leq_{X} \mathrm{~b}(y, \varepsilon) \stackrel{\text { def }}{\Longleftrightarrow} \rho(x, y)+\delta \leq \varepsilon, \\
& \mathrm{b}(x, \delta)<_{X} \mathrm{~b}(y, \varepsilon) \stackrel{\text { def }}{\Longleftrightarrow} \rho(x, y)+\delta<\varepsilon .
\end{aligned}
$$

The localic completion of $X$ is a formal topology $\mathcal{M}(X)=\left(M_{X}, \triangleleft_{X}, \leq_{X}\right)$ inductively generated by an axiom-set on $M_{X}$ consisting of the following axioms:

(M1) $a \triangleleft_{X}\left\{b \in M_{X} \mid b<_{X} a\right\}$,

(M2) $M_{X} \triangleleft_{X} \mathcal{C}_{\varepsilon}$ for each $\varepsilon \in \mathbb{Q}^{>0}$,

where $\mathcal{C}_{\varepsilon} \stackrel{\text { def }}{=}\left\{\mathrm{b}(x, \varepsilon) \in M_{X} \mid x \in X\right\}$ is the set of formal balls with radius $\varepsilon$.

Note that for any metric space $X$, its localic completion $\mathcal{M}(X)$ is always overt. This follows from the fact that for each axiom $a \triangleleft_{X} U$, the subset $U$ is inhabited. The localic completion of a metric space $X$ is always regular, and we have $a<_{X} b \Longrightarrow a \lll b$ for all $a, b \in M_{X}$; see Palmgren [14, Theorem 3.7].

For each metric space $X=(X, \rho)$, there exists a bijection $\varphi: \operatorname{Pt}(\mathcal{M}(X)) \rightarrow \widetilde{X}$ between the formal points of $\mathcal{M}(X)$ and the completion $\widetilde{X}$ of $X$ with Cauchy sequences. Then, $\operatorname{Pt}(\mathcal{M}(X))$ is given the induced metric $\rho_{\mathrm{Pt}}=\widetilde{\rho} \circ(\varphi \times \varphi)$, where $\widetilde{\rho}$ is the metric on $\widetilde{X}$. In 
this way, $\operatorname{Pt}(\mathcal{M}(X))$ is metrically identified with the completion of $X$; see Palmgren [14, Theorem 2.4]. There exists a dense isometry $i_{X}: X \rightarrow \operatorname{Pt}(\mathcal{M}(X))$ given by

$$
i_{X}(x) \stackrel{\text { def }}{=}\left\{\mathrm{b}(y, \varepsilon) \in M_{X} \mid \rho(x, y)<\varepsilon\right\},
$$

which is a metric isomorphism if and only if $X$ is complete.

Each formal ball $\mathrm{b}(x, \varepsilon)$ is associated with an open ball

$$
\mathrm{b}(x, \varepsilon)_{*} \stackrel{\text { def }}{=} B(x, \varepsilon) \stackrel{\text { def }}{=}\{y \in X \mid \rho(x, y)<\varepsilon\} .
$$

Dually, each $x \in X$ is associated with the set $\diamond x$ of open neighbourhoods of $x$, namely

$$
\diamond x \stackrel{\text { def }}{=}\left\{a \in M_{X} \mid x \in a_{*}\right\} .
$$

Note that $i_{X}(x)=\diamond x$. For any subset $Y \subseteq X$, we define $\diamond Y \stackrel{\text { def }}{=} \bigcup_{y \in Y} \diamond y$.

Example 3.2 (Palmgren [14, Example 2.2]) The localic completion of the rationals $\mathbb{Q}$ is the formal reals $\mathcal{R}$. To see this, let $d$ be the standard metric on $\mathbb{Q}$ given by $d(p, q)=|p-q|$. Then, the set $M_{\mathbb{Q}}$ of formal balls with the relations $\leq_{\mathbb{Q}}$ and $<_{\mathbb{Q}}$ is isomorphic (in the obvious sense) to the underlying structure $\left(S_{\mathcal{R}}, \leq_{\mathcal{R}},<_{\mathcal{R}}\right)$ of the formal reals $\mathcal{R}$, where $<_{\mathcal{R}}$ is defined by $(r, s)<_{\mathcal{R}}(p, q) \stackrel{\text { def }}{\Longleftrightarrow} p<r \& s<q$. Then, $\mathcal{M}(\mathbb{Q})$ is defined by the following axiom-set on $S_{\mathcal{R}}$ :

(Q1) $(p, q) \triangleleft_{\mathbb{Q}}\left\{(r, s) \in S_{\mathcal{R}} \mid(r, s)<_{\mathcal{R}}(p, q)\right\}$,

(Q2) $(p, q) \triangleleft \mathbb{Q} \mathcal{C}_{\varepsilon}$ for each $\varepsilon \in \mathbb{Q}^{>0}$.

Since the axioms (Q1) and (R1) are the same, it suffices to show that the axioms (Q2) and $(\mathrm{R} 2)$ are derivable in $\mathcal{R}$ and $\mathcal{M}(\mathbb{Q})$, respectively. First, assume $(\mathrm{R} 2)$. Let $(p, q)$ and $\varepsilon \in \mathbb{Q}^{>0}$. Then, by (R2), we have

$$
(p, q) \triangleleft_{\mathcal{R}}\{(p,(p+2 q) / 3),((2 p+q) / 3, q)\} .
$$

By applying this process sufficiently many times to each element of the set on the right hand side, we obtain $U \in \operatorname{Fin}\left(S_{\mathcal{R}}\right)$ such that $(p, q) \triangleleft_{\mathcal{R}} U$ and $s-r<\varepsilon$ for each $(r, s) \in U$. Hence $(p, q) \triangleleft_{\mathcal{R}} \mathcal{C}_{\varepsilon}$. Conversely, assume (Q2), and let $p, q, r, s \in \mathbb{Q}$ be such that $p<r<s<q$. Choose $\varepsilon \in \mathbb{Q}^{>0}$ such that $\varepsilon<s-r$. Then, by (Loc), we have $(p, q) \triangleleft_{\mathbb{Q}} \mathcal{C}_{\varepsilon} \downarrow(p, q) \leq_{\mathcal{R}}\{(p, s),(r, q)\}$, so $(p, q) \triangleleft_{\mathbb{Q}}\{(p, s),(r, q)\}$ as required.

It follows from the above observation that, assuming Countable Choice, the formal points of the formal reals $\mathcal{R}$, the Dedekind cuts, is metrically isomorphic to the Cauchy completion of $\mathbb{Q}$, namely the Cauchy reals. 
The following is crucial to our main result.

Theorem 3.3 (Palmgren [14, Theorem 2.7]) Let $X$ be a metric space, and let $Y \subseteq X$ be a dense subset of $X$. Then, $\mathcal{M}(Y) \cong \mathcal{M}(X)$.

In particular, the localic completion of a separable metric space is isomorphic to a formal topology $\mathcal{S}$ with a countable base set $S$. For example, the localic completion of the rationals $\mathbb{Q}$ and the reals $\mathbb{R}$ are isomorphic, ie $\mathcal{R} \cong \mathcal{M}(\mathbb{R}) \cong \mathcal{M}(\mathbb{Q})$.

\subsection{Functorial embedding}

We review the relevant facts about the functorial embedding of locally compact metric spaces into formal topologies established by Palmgren [14, Section 4 and Section 5].

Definition 3.4 (cf Bishop [2, Chapter 4]) A metric space $X=(X, \rho)$ is totally bounded if for each $\varepsilon \in \mathbb{Q}^{>0}$, there exists $Y=\left\{x_{0}, \ldots, x_{n-1}\right\} \in \operatorname{Fin}(X)$ such that for any $x \in X$, there exists $k<n$ such that $\rho\left(x, x_{k}\right)<\varepsilon$. Such a set $Y$ is called an $\varepsilon$-net. A metric space is compact if it is complete and totally bounded.

A metric space $X$ is locally compact if each open ball $B(x, \varepsilon)$ is contained in a compact subset of $X$. Thus, every compact metric space is locally compact. Every locally compact metric space is complete.

A function $f: X \rightarrow Y$ between locally compact metric spaces $(X, \rho)$ and $(Y, d)$ is said to be continuous if it is uniformly continuous on each open ball of $X$. If $X$ is compact, then a continuous function is just a uniformly continuous function. The locally compact metric spaces and continuous functions form a category LComp.

Remark 3.5 Bishop [2] defined a metric space to be totally bounded if for each $\varepsilon \in \mathbb{Q}^{>0}$ there exists an inhabited $\varepsilon$-net. Moreover, he required every locally compact metric space to be inhabited. Following Spitters [16], we drop these requirements. The results by Palmgren [14] on which our work depends still hold for our definitions.

The following three theorems play key roles in our main result.

Theorem 3.6 (Palmgren [14, Theorem 4.19]) If $X$ is a locally compact metric space, then $\mathcal{M}(X)$ is locally compact. In this case, $a<_{X} b \Longrightarrow a \ll b$ for all $a, b \in M_{X}$.

Remark 3.7 For a locally compact metric space $X$, the cover of $\mathcal{M}(X)$ admits a direct description in terms of the structure of $X$ (see Palmgren [14, Theorem 4.17]). Hence, our results about locally compact metric spaces do not require REA. 
Theorem 3.8 (Palmgren [14, Theorem 4.20]) If $X$ is a complete metric space, then $\mathcal{M}(X)$ is compact if and only if $X$ is totally bounded. ${ }^{3}$

Theorem 3.9 (Palmgren [14, Theorem 5.8]) The localic completion extends to a full and faithful functor $\mathcal{M}:$ LComp $\rightarrow$ FTop.

Here, we used the same symbol for localic completions and the functor $\mathcal{M}: \mathbf{L C o m p} \rightarrow$ FTop induced by them.

The functor $\mathcal{M}$ sends a continuous function $f: X \rightarrow Y$ in LComp to a formal topology map $\mathcal{M}(f): \mathcal{M}(X) \rightarrow \mathcal{M}(Y)$ defined by

$$
a \mathcal{M}(f) b \stackrel{\text { def }}{\Longleftrightarrow}\left(\exists c<_{Y} b\right) f\left[a_{*}\right] \subseteq c_{*}
$$

for all $a \in M_{X}$ and $b \in M_{Y}$, where $f\left[a_{*}\right]$ is the direct image of the subset $a_{*}$ under $f$.

Since $\mathcal{M}$ is full and faithful, $\mathcal{M}$ gives rise to an equivalence between LComp and its image category, denoted by $\mathcal{M}(\mathbf{L C o m p})$. The objects of $\mathcal{M}(\mathbf{L C o m p})$ is those of LComp (but denoted by $\mathcal{M}(X)$ for $X \in \mathbf{L C o m p}$ ) and the morphisms are formal topology maps between the localic completions of its domain and codomain. The quasi-inverse of $\mathcal{M}$, denoted by Pt: $\mathcal{M}(\mathbf{L C O m p}) \rightarrow$ LComp, sends an object $\mathcal{M}(X)$ (with the underlying locally compact metric space $(X, \rho))$ to a Bishop locally compact metric space $\operatorname{Pt}(\mathcal{M}(X))=\left(\operatorname{Pt}(\mathcal{M}(X)), \rho_{\mathrm{Pt}}\right)$, and Pt sends a formal topology map $r: \mathcal{M}(X) \rightarrow \mathcal{M}(Y)$ to a continuous function (in the sense of Definition 3.4) $\operatorname{Pt}(r): \operatorname{Pt}(\mathcal{M}(X)) \rightarrow \operatorname{Pt}(\mathcal{M}(Y))$ defined by

$$
\operatorname{Pt}(r)(\alpha) \stackrel{\text { def }}{=} r \alpha=\left\{b \in M_{Y} \mid(\exists a \in \alpha) a r b\right\} .
$$

For a locally compact metric space $X$, the function $i_{X}: X \rightarrow \operatorname{Pt}(\mathcal{M}(X))$ is an isomorphism since $X$ is complete. In fact, the family $\left(i_{X}: X \rightarrow \operatorname{Pt}(\mathcal{M}(X))\right)_{X \in \mathbf{L C o m p}}$ forms one of the natural isomorphisms of the equivalence (see Palmgren [14, Theorem 5.7]).

\section{Compact overt subtopologies}

The goal of this section is to show that, up to isomorphism, the localic completion induces a bijection between the compact subspaces of a locally compact metric space $X$ and the compact overt subtopologies of $\mathcal{M}(X)$. The results in this section refine and extend those of Spitters [16] and Coquand et al. [5].

We begin with the closed subsets of a metric space.

\footnotetext{
${ }^{3}$ Since every metric space can be seen as a dense subspace of its completion, the assumption that $X$ is complete is redundant.
} 
Definition 4.1 A subset $Y$ of a metric space $X$ is closed if for each $x \in X$, we have

$$
\left(\forall \varepsilon \in \mathbb{Q}^{>0}\right) B(x, \varepsilon) \curlyvee Y \Longrightarrow x \in Y .
$$

In terms of localic completion, $Y$ is closed in $X$ if and only if for any $x \in X$

$$
\diamond x \subseteq \diamond Y \Longrightarrow x \in Y .
$$

The class of closed subsets of a metric space $X$ is denoted by $\mathrm{Cl}(X)$.

The following generalises the result by Coquand et al. [5, Lemma 3.2], which can be obtained by Lemma 4.2 and Proposition 2.18. The proof requires Dependent Choice.

Lemma 4.2 Let $X=(X, \rho)$ be a metric space, and let Pos be a formal closed subset of $\mathcal{M}(X)$. Then, for each $a \in \operatorname{Pos}$, there exists $\alpha \in \operatorname{Pt}\left(\mathcal{M}(X)_{\text {Pos }}\right)$ such that $a \in \alpha$.

Proof Define a relation $R \subseteq \operatorname{Pos} \times$ Pos by

$$
\mathrm{b}(x, \varepsilon) R \mathrm{~b}(y, \delta) \stackrel{\text { def }}{\Longleftrightarrow} \mathrm{b}(y, \delta)<_{X} \mathrm{~b}(x, \varepsilon) \& \delta \leq \varepsilon / 2 .
$$

We show that $R$ is a total relation on Pos. Let $a=\mathrm{b}(x, \varepsilon) \in$ Pos. By (M1), (M2) and (Loc), we have

$$
a \triangleleft_{X}\left\{b \in M_{X} \mid b<_{X} a\right\} \downarrow \mathcal{C}_{\varepsilon / 2} .
$$

Since Pos $(a)$ and Pos is formal closed, there exists $b \in \operatorname{Pos}$ such that $b<_{X} a$ and $b \leq_{X} c$ for some $c \in \mathcal{C}_{\varepsilon / 2}$. Clearly, we have $a R b$, and hence $R$ is a total relation.

Let $a_{0} \in$ Pos. By Dependent Choice, there exists a function $f: \mathbb{N} \rightarrow$ Pos such that $f(0)=a_{0}$ and $f(n) R f(n+1)$ for all $n \in \mathbb{N}$. Put

$$
\alpha \stackrel{\text { def }}{=}\left\{a \in M_{X} \mid(\exists n \in \mathbb{N}) f(n) \leq_{X} a\right\} .
$$

Then $a_{0} \in \alpha$, and since Pos is upward closed, we have $\alpha \subseteq$ Pos. Moreover, it is easy to show that $\alpha$ is a formal point of $\mathcal{M}(X)$. Therefore, $\alpha \in \operatorname{Pt}\left(\mathcal{M}(X)_{\text {Pos }}\right)$.

Theorem 4.3 Let $X=(X, \rho)$ be a complete metric space. Then, there exists a bijection $\varphi: \operatorname{Cl}(X) \rightarrow \operatorname{Red}(\mathcal{M}(X))$ between the closed subsets of $X$ and the formal closed subsets of $\mathcal{M}(X)$ defined by

$$
\begin{aligned}
\varphi(Y) & \stackrel{\text { def }}{=} \diamond Y, \\
\varphi^{-1}(\mathrm{Pos}) & \stackrel{\text { def }}{=}\{x \in X \mid \diamond x \subseteq \operatorname{Pos}\} .
\end{aligned}
$$

Journal of Logic \& Analysis 9:5 (2017) 
Proof First, we show that for each $Y \in \mathrm{Cl}(X)$, the set $\diamond Y$ is formal closed. Let $a \in M_{X}$ and $U \subseteq M_{X}$, and suppose that $a \triangleleft_{X} U$ and $a \in \diamond Y$. Then, there exists $y \in Y$ such that $a \in \diamond_{y}$. Since $\diamond y \in \operatorname{Pt}(\mathcal{M}(X))$, we have $\diamond_{y}{ }_{\ell} U$, and hence $\diamond Y Y_{\ell} U$. Thus $\diamond Y$ is formal closed.

Next, we show that for each Pos $\in \operatorname{Red}(\mathcal{M}(X))$, the subset $Y=\{x \in X \mid \diamond x \subseteq \operatorname{Pos}\}$ is closed. Let $x \in X$, and suppose that $\diamond x \subseteq \diamond Y$. Let $a \in \diamond x$. Then, $a \in \diamond Y$, so there exists $y \in Y$ such that $a \in \diamond y$. Hence, $\diamond x \subseteq$ Pos, that is $x \in Y$. Therefore, $Y$ is a closed subset of $X$.

Lastly, we show that $\varphi$ is a bijection. The inclusion $Y \subseteq\left(\varphi^{-1} \circ \varphi\right)(Y)$ is obvious. The converse inclusion follows from the fact that $Y$ is closed. Hence $Y=\left(\varphi^{-1} \circ \varphi\right)(Y)$. Also, the inclusion $\left(\varphi \circ \varphi^{-1}\right)(\mathrm{Pos}) \subseteq$ Pos is clear. For the converse, let $a \in$ Pos. By Lemma 4.2, there exists $\alpha \in \operatorname{Pt}(\mathcal{M}(X))$ such that $a \in \alpha \subseteq$ Pos. Since $X$ is complete, there exists $x \in X$ such that $\diamond x=\alpha$. Thus, $a \in \diamond x$ and $x \in \varphi^{-1}$ (Pos). Hence $a \in\left(\varphi \circ \varphi^{-1}\right)(\mathrm{Pos})$, and therefore Pos $\subseteq\left(\varphi \circ \varphi^{-1}\right)(\mathrm{Pos})$.

Note that for any metric space $X$ and $\operatorname{Pos} \in \operatorname{Red}(\mathcal{M}(X))$, we have

$$
\varphi^{-1}(\operatorname{Pos})=i_{X}{ }^{-1}\left[\operatorname{Pt}\left(\mathcal{M}(X)_{\mathrm{Pos}}\right)\right] .
$$

Now we recall one of the most important notions in constructive topology.

Definition 4.4 A subset $Y$ of a metric space $X=(X, \rho)$ is located if for each $x \in X$ the distance $\rho(x, Y) \stackrel{\text { def }}{=} \inf \{\rho(x, y) \mid y \in Y\}$ exists as an extended Dedekind real number, ie for each $x \in X$, the subset

$$
U_{x}=\left\{q \in \mathbb{Q}^{>0} \mid(\exists y \in Y) \rho(x, y)<q\right\}
$$

satisfies

$$
\left(\forall p, q \in \mathbb{Q}^{>0}\right) p<q \Longrightarrow p \in \neg U_{x} \vee q \in U_{x}
$$

Remark 4.5 In the usual definition of located subset [2, Chapter 4, Section 2], the distance $\rho(x, Y)$ is required to be a Dedekind real, ie the subset $U_{x}$ is also required to be inhabited. We opted to drop this condition in order to obtain a smoother correspondence between the point-set and the point-free notions.

Lemma 4.6 A subset $Y \subseteq X$ of a metric space $X=(X, \rho)$ is located if and only if

$$
\left(\forall a, b \in M_{X}\right) a<_{X} b \Longrightarrow a \in \neg \diamond Y \vee b \in \diamond Y .
$$


Proof Suppose that $Y$ is located. Let $a, b \in M_{X}$ such that $a<_{X} b$, and write $a=\mathrm{b}(x, \varepsilon)$ and $b=\mathrm{b}(y, \delta)$. Choose $\gamma \in \mathbb{Q}^{>0}$ such that $\rho(x, y)+\varepsilon+\gamma<\delta$. Then, either $\varepsilon \in \neg U_{x}$ or $\varepsilon+\gamma \in U_{x}$. In the former case, we have $\neg[B(x, \varepsilon) \chi Y]$. In the latter case, we have $B(x, \varepsilon+\gamma) \chi Y$, and hence $B(y, \delta) \chi Y$. Therefore $a \in \neg \diamond Y$ or $b \in \diamond Y$.

Conversely, suppose that $Y$ satisfies the condition (4-2). Let $x \in X$ and let $p, q \in \mathbb{Q}^{>0}$ such that $p<q$. Then, $\mathrm{b}(x, p)<_{X} \mathrm{~b}(x, q)$. Hence, $\mathrm{b}(x, p) \in \neg \diamond Y$ or $\mathrm{b}(x, q) \in \diamond Y$, that is, $p \in \neg U_{x}$ or $q \in U_{x}$. Therefore, $Y$ is located.

Definition 4.7 (Spitters [16, Definition 44]) Let $\mathcal{M}(X)$ be the localic completion of a metric space $X$. A subset Pos $\subseteq M_{X}$ is called a located predicate on $\mathcal{M}(X)$ if Pos is formal closed and satisfies

$$
a<_{X} b \Longrightarrow a \in \neg \operatorname{Pos} \vee b \in \operatorname{Pos}
$$

for all $a, b \in M_{X}$. A subtopology $\mathcal{S}^{\prime}$ of $\mathcal{M}(X)$ is located if there exists a (necessarily unique) located predicate Pos on $\mathcal{M}(X)$ such that $\mathcal{S}^{\prime}=\mathcal{M}(X)_{\text {Pos }}$, where $\mathcal{M}(X)_{\text {Pos }}$ is the overt weakly closed subtopology of $\mathcal{M}(X)$ determined by Pos.

Spitters [16, Definition 44] defined a located subtopology of $\mathcal{M}(X)$ as a closed subtopology $\mathcal{M}(X)^{\mathcal{M}(X)-\neg \text { Pos }}$ determined by some located predicate Pos on $\mathcal{M}(X)$. However, the two definitions are equivalent.

Proposition 4.8 (Spitters [16, Proposition 51]) Let $X$ be a metric space and Pos be a located predicate on $\mathcal{M}(X)$. Then, $\mathcal{M}(X)^{\mathcal{M}(X)-\neg \text { Pos }}=\mathcal{M}(X)_{\text {Pos }}$.

Proof By Proposition 2.14, it suffices to show that $a \triangleleft_{X} \neg \operatorname{Pos} \cup(\operatorname{Pos} \cap\{a\})$ for each $a \in M_{X}$. Let $a \in M_{X}$, and let $b<_{X} a$. Since Pos is located, either $b \in \neg$ Pos or $\operatorname{Pos}(a)$. Since $b \triangleleft_{X} a$, we have $b \triangleleft_{X} \neg \operatorname{Pos} \cup(\operatorname{Pos} \cap\{a\})$. By (M1), we have $a \triangleleft_{X} \neg \operatorname{Pos} \cup(\operatorname{Pos} \cap\{a\})$.

For a metric space $X$, write $\operatorname{LCl}(X)$ for the class of closed located subsets of $X$ and $\operatorname{LRed}(\mathcal{M}(X))$ for the class of located predicates on $\mathcal{M}(X)$.

Theorem 4.9 Let $X$ be a complete metric space. The bijection $\varphi: \mathrm{Cl}(X) \rightarrow$ $\operatorname{Red}(\mathcal{M}(X))$ in Theorem 4.3 restricts to a bijection $\varphi: \operatorname{LCl}(X) \rightarrow \operatorname{LRed}(\mathcal{M}(X))$.

Proof For any $Y \in \operatorname{LCl}(X)$, the subset $\varphi(Y)$ is located by Lemma 4.6.

Conversely, let $\operatorname{Pos} \in \operatorname{LRed}(\mathcal{M}(X))$. Let $a, b \in M_{X}$ such that $a<_{X} b$. Since Pos is located, either $a \in \neg$ Pos or $b \in \operatorname{Pos}$, that is, either $a \in \neg \diamond \varphi^{-1}$ (Pos) or $b \in \diamond \varphi^{-1}(\mathrm{Pos})$. Thus, $\varphi^{-1}(\mathrm{Pos})$ is located by Lemma 4.6. 
Next, we define the notion of located subset for locally compact formal topologies. Definition 4.10 below extends the corresponding notion for compact regular formal topologies by Spitters [16, Definition 63], and it enjoys the similar characteristic property (see Theorem 4.13).

Definition 4.10 Let $\mathcal{S}$ be a locally compact formal topology. A subset Pos $\subseteq S$ is called a located predicate on $\mathcal{S}$ if Pos is formal closed and satisfies

$$
a \ll b \Longrightarrow a \in \neg \operatorname{Pos} \vee b \in \operatorname{Pos}
$$

for all $a, b \in S$. A subtopology $\mathcal{S}^{\prime}$ of a locally compact formal topology $\mathcal{S}$ is located if there exists a located predicate Pos on $\mathcal{S}$ such that $\mathcal{S}^{\prime}=\mathcal{S}^{\mathcal{S}-\neg \text { Pos }}$, ie $\mathcal{S}^{\prime}$ coincides with the closed subtopology determined by $\neg$ Pos.

Proposition 4.11 Let Pos $\subseteq S$ be a formal closed subset of a locally compact formal topology $\mathcal{S}$, and let wb: $S \rightarrow \operatorname{Pow}(S)$ be a function which makes $\mathcal{S}$ locally compact. Then, the following are equivalent:

(1) Pos is located;

(2) $a \in \mathrm{wb}(b) \Longrightarrow a \in \neg \operatorname{Pos} \vee b \in \operatorname{Pos}$ for all $a, b \in S$;

(3) $a \triangleleft \neg \operatorname{Pos} \cup(\operatorname{Pos} \cap\{a\})$ for all $a \in S$.

Proof (1) $\rightarrow(2)$ : Immediate from the fact that $a \in \mathrm{wb}(b) \Longrightarrow a \ll b$.

$(2) \rightarrow(3)$ : The proof is similar to that of Proposition 4.8. Note that $a \triangleleft \operatorname{wb}(a)$.

(3) $\rightarrow$ (1): Assume (3). Let $a, b \in S$ such that $a \ll b$. Since $b \triangleleft \neg \operatorname{Pos} \cup(\operatorname{Pos} \cap\{b\})$, there exists $U \in \operatorname{Fin}(\neg \operatorname{Pos} \cup(\operatorname{Pos} \cap\{b\}))$ such that $a \triangleleft U$. Either $U \subseteq \neg \operatorname{Pos}$ or $\operatorname{Pos}(b)$. In the former case, $\operatorname{Pos}(a)$ implies $\operatorname{Pos} \gamma \neg \operatorname{Pos}$, a contradiction. Hence $a \in \neg$ Pos. Therefore Pos is located.

By Theorem 3.6 and the axiom (M1), Definition 4.10 is compatible with Definition 4.7.

Corollary 4.12 Let $X$ be a locally compact metric space, and let Pos be a formal closed subset of $\mathcal{M}(X)$. Then, Pos is located with respect to $<_{X}$ if and only if it is located with respect to the way below relation $\ll$.

By Proposition 2.14 and Proposition 4.11, located subsets and overt closed subtopologies are equivalent in locally compact formal topologies. 
Theorem 4.13 Let $\mathcal{S}$ be a locally compact formal topology. Then, there exists an order preserving bijection Pos $\mapsto \mathcal{S}^{\mathcal{S}-\neg \text { Pos }}$ between the located predicates on $\mathcal{S}$ and the overt closed subtopologies of $\mathcal{S}$.

When specialised to compact regular formal topologies, Theorem 4.13 yields the following correspondence (cf Spitters [16, Theorem 74]).

Corollary 4.14 Let $\mathcal{S}$ be a compact regular formal topology. Then, there exists an order preserving bijection between the compact overt subtopologies of $\mathcal{S}$ and the located predicates on $\mathcal{S}$.

Proof Immediate from Proposition 2.21, Proposition 2.23 and Theorem 4.13.

We specialise the bijection of Theorem 4.9 to the compact subsets of a locally compact metric spaces. The following result is by Coquand et al. [5, Theorem 3.5]. We repeat their proof for the convenience of the reader.

Lemma 4.15 Let $X=(X, \rho)$ be a locally compact metric space. Let $\mathcal{S}_{\text {Pos }}$ be a compact overt subtopology of $\mathcal{M}(X)$ with a positivity Pos. Then, $Y=\varphi^{-1}(\operatorname{Pos})=$ $\{x \in X \mid \diamond x \subseteq$ Pos $\}$ is a compact subset of $X$.

Proof Let $\varepsilon \in \mathbb{Q}^{>0}$. By (M2) and compactness of $\mathcal{S}_{\text {Pos }}$, there exist $x_{0}, \ldots, x_{n-1} \in X$ such that $M_{X} \triangleleft \operatorname{Pos}\left\{\mathrm{b}\left(x_{i}, \varepsilon / 2\right) \mid i<n\right\} \subseteq$ Pos. Let $i<n$. By Lemma 4.2, there exists $\alpha_{i} \in \operatorname{Pt}(\mathcal{M}(X))$ such that $\mathrm{b}\left(x_{i}, \varepsilon / 2\right) \in \alpha_{i} \subseteq \operatorname{Pos}$, and since $X$ is complete, there exists $y_{i} \in X$ such that $\diamond y_{i}=\alpha_{i}$. Put $Y_{\varepsilon}=\left\{y_{i} \mid i<n\right\}$. Note that $Y_{\varepsilon}$ is a subset of $Y$. Let $y \in Y$. Since $\diamond y \in \operatorname{Pt}\left(\mathcal{M}(X)_{\text {Pos }}\right)$, there exists $i<n$ such that $\mathrm{b}\left(x_{i}, \varepsilon / 2\right) \in \diamond y$. Then, $\rho\left(y_{i}, y\right)<\varepsilon$. Hence, $Y_{\varepsilon}$ is an $\varepsilon$-net, so $Y$ is totally bounded. Since $Y$ is closed and $X$ is complete, $Y$ is also complete. Therefore, $Y$ is metrically compact.

Theorem 4.16 Let $X=(X, \rho)$ be a locally compact metric space. Then, up to isomorphism, the localic completion induces a bijection between the compact subspaces of $X$ and the compact overt subtopologies of $\mathcal{M}(X)$.

Proof We will identify a compact subspace of $X$ with a compact subset of $X$. We define a bijection $\Phi$ and its inverse $\Phi^{-1}$ between the compact subsets of $X$ and the compact overt subtopologies of $\mathcal{M}(X)$ such that

$$
\begin{aligned}
\Phi(Y) & \cong \mathcal{M}(Y), \\
\Phi^{-1}\left(\mathcal{S}_{\text {Pos }}\right) & \cong \operatorname{Pt}\left(\mathcal{S}_{\text {Pos }}\right)
\end{aligned}
$$


for any compact subset $Y \subseteq X$ and for any compact overt subtopology $\mathcal{S}_{\text {Pos }} \sqsubseteq \mathcal{M}(X)$ with a positivity predicate Pos.

First, given a compact subset $Y \subseteq X$, let $i_{Y}: Y \rightarrow X$ be the inclusion. Let $\Phi(Y)$ be the image of $\mathcal{M}(Y)$ under the embedding $\mathcal{M}\left(i_{Y}\right): \mathcal{M}(Y) \rightarrow \mathcal{M}(X)$. Note that $\mathcal{M}\left(i_{Y}\right)$ is defined by

$$
a \mathcal{M}\left(i_{Y}\right) b \stackrel{\text { def }}{\Longleftrightarrow}\left(\exists b^{\prime}<_{X} b\right) i_{Y}\left[a_{*}\right] \subseteq b_{*}^{\prime}
$$

for all $a \in M_{Y}$ and $b \in M_{X}$. Since $\mathcal{M}(Y)$ is compact overt, $\Phi(Y)$ is a compact overt subtopology of $\mathcal{M}(X)$. Clearly, $\Phi(Y) \cong \mathcal{M}(Y)$.

Conversely, given a compact overt subtopology $\mathcal{S}_{\text {Pos }}$ of $\mathcal{M}(X)$ with a positivity Pos, let $\Phi^{-1}\left(\mathcal{S}_{\text {Pos }}\right)=i_{X}^{-1}\left[\operatorname{Pt}\left(\mathcal{S}_{\text {Pos }}\right)\right]=\{x \in X \mid \diamond x \subseteq$ Pos $\}$. By Lemma $4.15, \Phi^{-1}\left(\mathcal{S}_{\text {Pos }}\right)$ is a compact subset of $X$. Moreover, since $X$ is complete, we have $\Phi^{-1}\left(\mathcal{S}_{\text {Pos }}\right) \cong \operatorname{Pt}\left(\mathcal{S}_{\text {Pos }}\right)$.

To show that $\Phi$ and $\Phi^{-1}$ are inverse to each other, first, let $Y \subseteq X$ be a compact subset of $X$. Since $\mathcal{M}(Y)$ is compact overt with the positivity $M_{Y}, \Phi(Y)$ has a positivity $\operatorname{Pos}_{Y}=\mathcal{M}\left(i_{Y}\right) M_{Y}=\left\{a \in M_{X} \mid\left(\exists b \in M_{Y}\right) b \mathcal{M}\left(i_{Y}\right) a\right\}$ by Lemma 2.10. We show that $\operatorname{Pos}_{Y}=\diamond Y$. Let $a \in \operatorname{Pos}_{Y}$. Then, there exists $b \in M_{Y}$ such that $b \mathcal{M}\left(i_{Y}\right) a$. Clearly, we have $a \in \diamond Y$. Conversely, let $a=\mathrm{b}(x, \varepsilon) \in \diamond Y$. Then, there exists $y \in Y$ such that $\rho(x, y)<\varepsilon$. Choose $\delta \in \mathbb{Q}^{>0}$ such that $\rho(x, y)+\delta<\varepsilon$. Then, $\mathrm{b}(y, \delta)<_{X} a$, so $\mathrm{b}(y, \delta) \mathcal{M}\left(i_{Y}\right) a$. Hence, $a \in \operatorname{Pos}_{Y}$, and thus $\operatorname{Pos}_{Y}=\diamond Y$. Since $Y$ is a closed subset of $X$, we have $\left(\Phi^{-1} \circ \Phi\right)(Y)=\{x \in X \mid \diamond x \subseteq \diamond Y\}=Y$.

Conversely, let $\mathcal{S}_{\text {Pos }}$ be a compact overt subtopology of $\mathcal{M}(X)$ with a positivity Pos. Then, $\mathcal{S}_{\text {Pos }}$ is uniquely determined by the located predicate Pos. Moreover, $(\Phi \circ$ $\left.\Phi^{-1}\right)\left(\mathcal{S}_{\text {Pos }}\right)$ is uniquely determined by the positivity $\diamond \Phi^{-1}\left(\mathcal{S}_{\text {Pos }}\right)$. But $\diamond \Phi^{-1}\left(\mathcal{S}_{\text {Pos }}\right)=$ $\left(\varphi \circ \varphi^{-1}\right)($ Pos $)=$ Pos, where $\varphi$ is the bijection described in Theorem 4.3. Hence $\left(\Phi \circ \Phi^{-1}\right)\left(\mathcal{S}_{\text {Pos }}\right)=\mathcal{S}_{\text {Pos }}$.

Corollary 4.17 Let $\mathcal{S}$ be a formal topology. Then, the following are equivalent.

(1) $\mathcal{S}$ is isomorphic to the localic completion of some compact metric space.

(2) $\mathcal{S}$ is isomorphic to a compact overt subtopology of the localic completion of some locally compact metric space.

Example 4.18 The formal unit interval $\mathcal{I}[0,1]$ is isomorphic to the localic completion of the unit interval $[0,1]$. In fact, $[0,1]$ is a compact subspace of $\mathbb{R}$, which is locally compact. Hence, $\mathcal{M}([0,1])$ is isomorphic to a compact overt subtopology $\mathcal{S}$ of the formal reals $\mathcal{R}$ with a positivity Pos defined by

$$
\operatorname{Pos}=\diamond[0,1]=\left\{(p, q) \in S_{\mathcal{R}} \mid 0<q \& p<1\right\} .
$$


Since $\mathcal{S}$ is located, $\mathcal{S}$ coincides with the closed subtopology determined by

$$
\neg \operatorname{Pos}=\left\{(p, q) \in S_{\mathcal{R}} \mid q \leq 0 \vee 1 \leq p\right\}=_{\mathcal{R}}(-\infty, 0) \cup(1, \infty),
$$

which is exactly the formal unit interval $\mathcal{I}[0,1]$ (see Example 2.12).

\section{Localic completion of products}

We show that the functor $\mathcal{M}:$ LComp $\rightarrow$ FTop preserves countable products of inhabited compact metric spaces.

We first recall the construction of a product of inductively generated formal topologies from Vickers [18]. Let $\left(\mathcal{S}_{i}\right)_{i \in I}$ be a set-indexed family of inductively generated formal topologies, each of the form $\mathcal{S}_{i}=\left(S_{i}, \triangleleft_{i}, \leq_{i}\right)$, and let $I_{i}, C_{i}$ be the axiom-set which generates $\mathcal{S}_{i}$. Define a preorder $\left(S_{\Pi}, \leq_{\Pi}\right)$ on a set $S_{\Pi}=$ Fin $\left(\sum_{i \in I} S_{i}\right)$ by

$$
A \leq_{\Pi} B \stackrel{\text { def }}{\Longleftrightarrow}(\forall(i, b) \in B)(\exists(j, a) \in A) i=j \& a \leq_{i} b .
$$

The axiom-set on $S_{\Pi}$ is defined by

(S1) $S_{\Pi} \triangleleft \Pi\left\{\{(i, a)\} \in S_{\Pi} \mid a \in S_{i}\right\}$ for each $i \in I$,

(S2) $\{(i, a),(i, b)\} \triangleleft_{\Pi}\left\{\{(i, c)\} \in S_{\Pi} \mid c \leq_{i} a \& c \leq_{i} b\right\}$,

(S3) $\{(i, a)\} \triangleleft \Pi\left\{\{(i, b)\} \in S_{\Pi} \mid b \in C_{i}(a, k)\right\}$ for each $k \in I_{i}(a)$.

Let $\prod_{i \in I} \mathcal{S}_{i}=\left(S_{\Pi}, \triangleleft_{\Pi}, \leq_{\Pi}\right)$ denote the formal topology inductively generated by the above axiom-set. For each $i \in I$, the projection $p_{i}: \prod_{i \in I} \mathcal{S}_{i} \rightarrow \mathcal{S}_{i}$ is defined by

$$
A p_{i} a \stackrel{\text { def }}{\Longleftrightarrow} A=\{(i, a)\}
$$

for all $A \in S_{\Pi}$ and $a \in S_{i}$. Then, the family of projections $\left(p_{i}: \prod_{i \in I} \mathcal{S}_{i} \rightarrow \mathcal{S}_{i}\right)_{i \in I}$ is a product of $\left(\mathcal{S}_{i}\right)_{i \in I}$. Given a family $\left(r_{i}: \mathcal{S} \rightarrow \mathcal{S}_{i}\right)_{i \in I}$ of formal topology maps, we have a unique formal topology map $r: \mathcal{S} \rightarrow \prod_{i \in I} \mathcal{S}_{i}$ which commutes with each projection. The map $r$ is defined by

$$
\operatorname{arA} \stackrel{\text { def }}{\Longleftrightarrow}(\forall(i, b) \in A) a \triangleleft r_{i}^{-}\{b\}
$$

for all $a \in S$ and $A \in S_{\Pi}$.

Next, the product of a sequence $\left(X_{n}, \rho_{n}\right)_{n \in \mathbb{N}}$ of metric spaces is a set theoretic product $\prod_{n \in \mathbb{N}} X_{n}$ equipped with a metric $\rho$ defined by

$$
\rho\left(\left(x_{n}\right)_{n \in \mathbb{N}},\left(y_{n}\right)_{n \in \mathbb{N}}\right) \stackrel{\text { def }}{=} \sum_{n=0}^{\infty} \frac{\rho_{n}\left(x_{n}, y_{n}\right)}{2^{n+1}} .
$$


Here, we are assuming that each metric $\rho_{n}$ is bounded by 1 without loss of generality. Then, the family of projections $\pi_{n}: X \rightarrow X_{n}$ forms the product of the metric spaces. A countable product of inhabited compact metric spaces is compact; see Bishop [2, Chapter 4, Section 4, Proposition 6]. We write $\left(x_{n}\right)$ for the element $\left(x_{n}\right)_{n \in \mathbb{N}}$ of $\prod_{n \in \mathbb{N}} X_{n}$. Let $\left(X_{n}, \rho_{n}\right)_{n \in \mathbb{N}}$ be a sequence of inhabited compact metric spaces, and let $\prod_{n \in \mathbb{N}} \mathcal{M}\left(X_{n}\right)=\left(S_{\Pi}, \triangleleft_{\Pi}, \leq_{\Pi}\right)$ be the product of the localic completions $\left(\mathcal{M}\left(X_{n}\right)\right)_{n \in \mathbb{N}}$. Since $\mathcal{M}\left(X_{n}\right)$ is compact regular for each $n \in \mathbb{N}$ by Theorem 3.8, the product $\prod_{n \in \mathbb{N}} \mathcal{M}\left(X_{n}\right)$ is compact regular (see Cederquist and Coquand [4]).

Lemma 5.1 Let $r: \mathcal{M}\left(\prod_{n \in \mathbb{N}} X_{n}\right) \rightarrow \prod_{n \in \mathbb{N}} \mathcal{M}\left(X_{n}\right)$ be the unique formal topology map determined by the sequence $\left(\mathcal{M}\left(\pi_{n}\right): \mathcal{M}\left(\prod_{n \in \mathbb{N}} X_{n}\right) \rightarrow \mathcal{M}\left(X_{n}\right)\right)_{n \in \mathbb{N}}$ of formal topology maps. Then, $r$ is an embedding.

Proof Write $\mathcal{M}\left(\prod_{n \in \mathbb{N}} X_{n}\right)=\left(M_{X}, \triangleleft_{X}, \leq_{X}\right)$, and write $r_{i}$ for the localic completion $\mathcal{M}\left(\pi_{i}\right): \mathcal{M}\left(\prod_{n \in \mathbb{N}} X_{n}\right) \rightarrow \mathcal{M}\left(X_{i}\right)$ of the projection $\pi_{i}: \prod_{n \in \mathbb{N}} X_{n} \rightarrow X_{i}$.

Let $a=\mathrm{b}\left(\left(x_{n}\right), \varepsilon\right) \in M_{X}$. We must show that $a \triangleleft_{X} r^{-} r^{-*} \mathcal{A}_{X}\{a\}$. By (M1), we have $\mathrm{b}\left(\left(x_{n}\right), \varepsilon\right) \triangleleft_{X}\left\{\mathrm{~b}\left(\left(x_{n}\right), \varepsilon^{\prime}\right) \in M_{X} \mid \varepsilon^{\prime}<\varepsilon\right\}$.

Let $\varepsilon^{\prime}<\varepsilon$, and choose $\gamma \in \mathbb{Q}^{>0}$ such that $\varepsilon^{\prime}+3 \gamma<\varepsilon$. Choose a positive number $N \in \mathbb{N}$ and $\gamma^{\prime} \in \mathbb{Q}^{>0}$ such that $2^{-N}<\gamma^{\prime}<\gamma$. By (M2) and (Loc), we have

$$
\mathrm{b}\left(\left(x_{n}\right), \varepsilon^{\prime}\right) \triangleleft_{X} \mathcal{C}_{\gamma^{\prime} /\left(2^{N+1} N\right)} \downarrow \mathrm{b}\left(\left(x_{n}\right), \varepsilon^{\prime}\right) .
$$

Let $\mathrm{b}\left(\left(y_{n}\right), \theta\right) \in \mathcal{C}_{\gamma^{\prime} /\left(2^{N+1} N\right)} \downarrow \mathrm{b}\left(\left(x_{n}\right), \varepsilon^{\prime}\right)$. We show that $\mathrm{b}\left(\left(y_{n}\right), \theta\right) \in r^{-} r^{-*} \mathcal{A}_{X}\{a\}$. Define an element $A$ of $S_{\Pi}$ by $A=\left\{\left(i, \mathrm{~b}\left(y_{i}, \gamma / N\right)\right) \mid i<N\right\}$. Then, for each $i<N$, we have

$$
\pi_{i}\left[B\left(\left(y_{n}\right), \theta\right)\right] \subseteq B\left(y_{i}, \gamma^{\prime} / N\right) .
$$

Thus, $\mathrm{b}\left(\left(y_{n}\right), \theta\right) r_{i} \mathrm{~b}\left(y_{i}, \gamma / N\right)$ for each $i<N$. Hence $\mathrm{b}\left(\left(y_{n}\right), \theta\right) r A$. It remains to be shown that $r^{-}\{A\} \triangleleft_{X}\{a\}$. By (M2) and the definition of $r$, it suffices to show that

$$
r_{0}^{-}\left\{\mathrm{b}\left(y_{0}, \gamma / N\right)\right\} \downarrow \cdots \downarrow r_{N-1}^{-}\left\{\mathrm{b}\left(y_{N-1}, \gamma / N\right)\right\} \downarrow \mathcal{C}_{\gamma} \triangleleft_{X} a .
$$

Let $\mathrm{b}\left(\left(z_{n}\right), \delta\right) \in r_{0}^{-}\left\{\mathrm{b}\left(y_{0}, \gamma / N\right)\right\} \downarrow \cdots \downarrow r_{N-1}^{-}\left\{\mathrm{b}\left(y_{N-1}, \gamma / N\right)\right\} \downarrow \mathcal{C}_{\gamma}$. Then, we have $\delta \leq \gamma$ and $\rho_{i}\left(z_{i}, y_{i}\right)<\gamma / N$ for all $i<N$. Thus

$$
\begin{aligned}
\rho\left(\left(z_{n}\right),\left(x_{n}\right)\right)+\delta & \leq \rho\left(\left(z_{n}\right),\left(y_{n}\right)\right)+\rho\left(\left(y_{n}\right),\left(x_{n}\right)\right)+\gamma \\
& \leq \sum_{i=N}^{\infty} 2^{-(i+1)}+\sum_{i=0}^{N-1} \rho_{i}\left(z_{i}, y_{i}\right) / 2^{i+1}+\rho\left(\left(y_{n}\right),\left(x_{n}\right)\right)+\gamma \\
& \leq 3 \gamma+\rho\left(\left(y_{n}\right),\left(x_{n}\right)\right) \\
& <3 \gamma+\rho\left(\left(y_{n}\right),\left(x_{n}\right)\right)+\theta \\
& <3 \gamma+\varepsilon^{\prime}<\varepsilon .
\end{aligned}
$$


Hence, $\mathrm{b}\left(\left(z_{n}\right), \delta\right)<_{X} \mathrm{~b}\left(\left(x_{n}\right), \varepsilon\right)$, and thus $\mathrm{b}\left(\left(z_{n}\right), \delta\right) \triangleleft_{X} a$, as required.

The image $\mathcal{S}_{r}$ of $\mathcal{M}\left(\prod_{n \in \mathbb{N}} X_{n}\right)$ under $r$ is a compact overt subtopology of $\prod_{n \in \mathbb{N}} \mathcal{M}\left(X_{n}\right)$ with a positivity $\operatorname{Pos}_{X}=r M_{X}$.

Lemma 5.2 $\operatorname{Pos}_{X}$ is a positivity of $\prod_{n \in \mathbb{N}} \mathcal{M}\left(X_{n}\right)$.

Proof Write $\prod_{n \in \mathbb{N}} \mathcal{M}\left(X_{n}\right)=\left(S_{\Pi}, \triangleleft_{\Pi}, \leq_{\Pi}\right)$. We must show that $A \triangleleft_{\Pi} \operatorname{Pos}_{X} \cap\{A\}$ for all $A \in S_{\Pi}$. Let $A \in S_{\Pi}$. By the axiom (S2), there exists a subset $\mathcal{U} \subseteq S_{\Pi}$ such that $A \triangleleft_{\Pi} \mathcal{U}$ and each $A^{\prime} \in \mathcal{U}$ satisfies $A^{\prime} \leq_{\Pi} A$ and

$$
\left(\forall(n, a),(m, b) \in A^{\prime}\right) n=m \Longrightarrow a=b .
$$

Let $A^{\prime} \in \mathcal{U}$. We can write $A^{\prime}$ as $\left\{\left(n_{0}, a_{0}\right), \ldots,\left(n_{N-1}, a_{N-1}\right)\right\}$ such that $n_{i} \neq n_{j}$ for all $0 \leq i<j<N$. For each $i<N$, write $a_{i}=\mathrm{b}\left(x_{i}, \varepsilon_{i}\right)$.

Since each $X_{n}$ is inhabited, we may choose a sequence $\left(z_{n}\right) \in \prod_{n \in \mathbb{N}} X_{n}$ by Countable Choice. Define a sequence $\left(y_{n}\right) \in \prod_{n \in \mathbb{N}} X_{n}$ by

$$
y_{n}= \begin{cases}x_{i} & \text { if } n_{i}=n \text { for some } i<N \\ z_{n} & \text { otherwise }\end{cases}
$$

Choose $\delta \in \mathbb{Q}^{>0}$ such that $\delta<\min \left\{\varepsilon_{i} / 2^{n_{i}+1} \mid i<N\right\}$. Then, $\mathrm{b}\left(\left(y_{n}\right), \delta\right) r_{n_{i}} \mathrm{~b}\left(x_{i}, \varepsilon_{i}\right)$ for each $i<N$, and thus $\mathrm{b}\left(\left(y_{n}\right), \delta\right) r A^{\prime}$. Hence $A^{\prime} \in \operatorname{Pos}_{X}$. Since $\operatorname{Pos}_{X}$ is upward closed, we have $A \in \operatorname{Pos}_{X}$. Hence $A^{\prime} \triangleleft_{\Pi} \operatorname{Pos}_{X} \cap\{A\}$. Therefore $A \triangleleft_{\Pi} \operatorname{Pos}_{X} \cap\{A\}$.

Lemma 5.3 The embedding $r: \mathcal{M}\left(\prod_{n \in \mathbb{N}} X_{n}\right) \rightarrow \prod_{n \in \mathbb{N}} \mathcal{M}\left(X_{n}\right)$ is an isomorphism.

Proof Since $\prod_{n \in \mathbb{N}} \mathcal{M}\left(X_{n}\right)$ is regular, the image $\mathcal{S}_{r}$ is closed and overt by Proposition 2.21. Thus it is the largest subtopology of $\prod_{n \in \mathbb{N}} \mathcal{M}\left(X_{n}\right)$ with positivity $\operatorname{Pos}_{X}$ by Proposition 2.18. Hence $\mathcal{S}_{r}=\prod_{n \in \mathbb{N}} \mathcal{M}\left(X_{n}\right)$ by Lemma 5.2. Since $r$ is an embedding by Lemma $5.1, r$ is an isomorphism.

Theorem 5.4 The functor $\mathcal{M}:$ LComp $\rightarrow$ FTop preserves countable products of inhabited compact metric spaces. 


\section{The main result}

We begin with the predicative notion of complete regularity introduced by Curi [7].

Definition 6.1 Let $\mathbb{I}$ denote the set $\{q \in \mathbb{Q} \mid 0 \leq q \leq 1\}$. Given a formal topology $\mathcal{S}$ and subsets $U, V \subseteq S$, a scale from $U$ to $V$ is a family $\left(U_{q}\right)_{q \in \mathbb{I}}$ of subsets of $S$ such that $U \triangleleft U_{0}, U_{1} \triangleleft V$, and $p<q \Longrightarrow U_{p} \lll U_{q}$ for all $p, q \in \mathbb{I}$. For subsets $U, V \subseteq S$, we say that $U$ is really covered by $V$, written $U \nVdash V$, if there is a scale from $U$ to $V$.

Lemma 6.2 Let $r: \mathcal{S}^{\prime} \rightarrow \mathcal{S}$ be a formal topology map, and let $V \subseteq S$. Then, $r$ factors through the inclusion $\mathcal{S}^{\mathcal{S}-V} \rightarrow \mathcal{S}$ if and only if $r^{-} V \triangleleft^{\prime} \emptyset$.

Proof Let $\mathcal{S}_{r}$ denote the image of $\mathcal{S}^{\prime}$ under $r$. Then

$$
\begin{aligned}
r \text { factors through } \mathcal{S}^{\mathcal{S}-V} & \Longleftrightarrow \mathcal{S}_{r} \sqsubseteq \mathcal{S}^{\mathcal{S}-V} \\
& \Longleftrightarrow V \triangleleft_{r} \emptyset \\
& \Longleftrightarrow r^{-} V \triangleleft^{\prime} \emptyset .
\end{aligned}
$$

Let $\mathcal{R}$ be the formal reals (see Example 2.8). For each $q \in \mathbb{Q}$, define

$$
\begin{aligned}
(q, \infty) & \stackrel{\text { def }}{=}\left\{(r, s) \in S_{\mathcal{R}} \mid r=q\right\}, \\
(-\infty, q) & \stackrel{\text { def }}{=}\left\{(r, s) \in S_{\mathcal{R}} \mid s=q\right\} .
\end{aligned}
$$

In the following proposition, $\mathcal{I}[0,1]$ denotes the formal unit interval (see Example 2.12).

Proposition 6.3 (Johnstone [11, Chapter IV, Proposition 1.4]) Let $\mathcal{S}$ be a formal topology, and let $U, V \subseteq S$. Then, the following are equivalent.

(1) $U \nVdash V$.

(2) There exists a formal topology map $r: \mathcal{S} \rightarrow \mathcal{R}$ such that
(a) $r^{-}(0, \infty) \downarrow U \triangleleft \emptyset$,
(b) $r^{-}(-\infty, 1) \triangleleft V$.

(3) There exists a formal topology map as in (2), but additionally factors through $\mathcal{I}[0,1]$. 
Proof (2) $\rightarrow$ (1): Let $r: \mathcal{S} \rightarrow \mathcal{R}$ be a formal topology map which satisfies (2a) and (2b). Let $U_{0}=U$. For each $q \in \mathbb{I} \cap \mathbb{Q}^{>0}$, define

$$
U_{q} \stackrel{\text { def }}{=} r^{-}(-\infty, q)
$$

It is easy to show that $p<q$ implies $(-\infty, p) \lll(-\infty, q)$, and thus $p<q$ implies $r^{-}(-\infty, p) \lll r^{-}(-\infty, q)$. Hence $p<q \Longrightarrow U_{p} \lll U_{q}$ for each $p, q \in \mathbb{Q}$. Also, we have $U_{1} \triangleleft V$ by (2b). Moreover, for any $q \in \mathbb{I} \cap \mathbb{Q}^{>0}$, since $S_{\mathcal{R}} \triangleleft_{\mathcal{R}}(-\infty, q) \cup(0, \infty)$, we have $S \triangleleft r^{-}(-\infty, q) \cup r^{-}(0, \infty) \triangleleft U_{q} \cup U^{*}$ by $(2 \mathrm{a})$, and hence $U_{0} \lll U_{q}$. Therefore, $\left(U_{q}\right)_{q \in \mathbb{I}}$ is a scale from $U$ to $V$.

$(1) \rightarrow$ (3): Let $\left(U_{q}\right)_{q \in \mathbb{I}}$ be a scale from $U$ to $V$. Extend $\left(U_{q}\right)_{q \in \mathbb{I}}$ to $\left(U_{q}\right)_{q \in \mathbb{Q}}$ by defining $U_{q}=\emptyset$ if $q<0$, and $U_{q}=S$ if $1<q$. Then, we have $p<q \Longrightarrow U_{p} \lll U_{q}$. Define a relation $r \subseteq S \times S_{\mathcal{R}}$ by

$$
\operatorname{ar}(p, q) \stackrel{\text { def }}{\Longleftrightarrow}\left(\exists\left(p^{\prime}, q^{\prime}\right) \in S_{\mathcal{R}}\right) p<p^{\prime}<q^{\prime}<q \& a \triangleleft U_{p^{\prime}}^{*} \downarrow U_{q^{\prime}} .
$$

We show that $r$ is a formal topology map (see Remark 2.7). The conditions (FTM1) and (FTM3a) are easy to show. We check the other conditions:

(FTM2): Let $(p, q),(u, v) \in S_{\mathcal{R}}$, and let $a \in r^{-}\{(p, q)\} \downarrow r^{-}\{(u, v)\}$. Then, there exist $\left(p^{\prime}, q^{\prime}\right),\left(u^{\prime}, v^{\prime}\right) \in S_{\mathcal{R}}$ such that $p<p^{\prime}<q^{\prime}<q$ and $a \triangleleft U_{p^{\prime}}^{*} \downarrow U_{q^{\prime}}$, and $u<u^{\prime}<v^{\prime}<v$ and $a \triangleleft U_{u^{\prime}}^{*} \downarrow U_{v^{\prime}}$. Then

$$
a \triangleleft U_{p^{\prime}}^{*} \downarrow U_{u^{\prime}}^{*} \downarrow U_{q^{\prime}} \downarrow U_{v^{\prime}}=\mathcal{S} U_{\max \left(p^{\prime}, u^{\prime}\right)}^{*} \downarrow U_{\min \left(q^{\prime}, v^{\prime}\right)} .
$$

If $\max \left(p^{\prime}, u^{\prime}\right)<\min \left(q^{\prime}, v^{\prime}\right)$, then we have $a \triangleleft r^{-}((p, q) \downarrow(u, v))$. Otherwise, we must have $a \triangleleft \emptyset$ because $U_{p} \downarrow U_{q}^{*} \triangleleft U_{p}^{* *} \downarrow U_{q}^{*} \triangleleft U_{q}^{* *} \downarrow U_{q}^{*} \triangleleft \emptyset$ whenever $p \leq q$. Thus, in either case, we have $a \triangleleft r^{-}((p, q) \downarrow(u, v))$.

(FTM3b): It suffices to show that $r$ preserves (R1) and (R2). For (R2), let $(p, q),(u, v) \in$ $S_{\mathcal{R}}$ such that $p<u<v<q$, and let $a r(p, q)$. Then, there is $\left(p^{\prime}, q^{\prime}\right) \in S_{\mathcal{R}}$ such that $p<p^{\prime}<q^{\prime}<q$ and $a \triangleleft U_{p^{\prime}}^{*} \downarrow U_{q^{\prime}}$. If $q^{\prime}<v$ or $u<p^{\prime}$, then we immediately have $a \triangleleft r^{-}\{(p, v),(u, q)\}$. So suppose that $p^{\prime} \leq u<v \leq q^{\prime}$. Let $u^{\prime}, v^{\prime} \in \mathbb{Q}$ such that $u<u^{\prime}<v^{\prime}<v$. Then

$$
\begin{aligned}
\left(U_{p^{\prime}}^{*} \downarrow U_{v^{\prime}}\right) \cup\left(U_{u^{\prime}}^{*} \downarrow U_{q^{\prime}}\right) & =\mathcal{S}\left(U_{p^{\prime}}^{*} \cup U_{u^{\prime}}^{*}\right) \downarrow\left(U_{p^{\prime}}^{*} \cup U_{q^{\prime}}\right) \downarrow\left(U_{v^{\prime}} \cup U_{u^{\prime}}^{*}\right) \downarrow\left(U_{v^{\prime}} \cup U_{q^{\prime}}\right) \\
& =\mathcal{S}\left(U_{p^{\prime}}^{*} \cup U_{u^{\prime}}^{*}\right) \downarrow S \downarrow S \downarrow\left(U_{v^{\prime}} \cup U_{q^{\prime}}\right) \\
& =\mathcal{S} U_{p^{\prime}}^{*} \downarrow U_{q^{\prime}} .
\end{aligned}
$$

Thus,

$$
a \triangleleft\left(U_{p^{\prime}}^{*} \downarrow U_{v^{\prime}}\right) \cup\left(U_{u^{\prime}}^{*} \downarrow U_{q^{\prime}}\right) \triangleleft r^{-}\{(p, v)\} \cup r^{-}\{(u, q)\} \triangleleft r^{-}\{(p, v),(u, q)\} .
$$

Hence $r$ preserves (R2). It is also easy to see that $r$ preserves (R1). 
Next, we show that $r$ satisfies (2a) and (2b). The condition (2b) is immediate from the fact that $q<1$ implies $U_{q} \triangleleft V$. As for the condition (2a), we have

$$
r^{-}(0, \infty) \downarrow U \triangleleft\left(\bigcup_{q \in \mathbb{Q}^{>0}}\left(U_{0}^{*} \downarrow U_{q}\right)\right) \downarrow U_{0}=\mathcal{S} \bigcup_{q \in \mathbb{Q}^{>0}}\left(U_{0}^{*} \downarrow U_{q} \downarrow U_{0}\right) \triangleleft \emptyset .
$$

Lastly, $r$ factors through $\mathcal{I}[0,1]$ by Lemma 6.2, since we have

$$
r^{-}((-\infty, 0) \cup(1, \infty)) \triangleleft r^{-}(-\infty, 0) \cup r^{-}(1, \infty) \triangleleft \emptyset
$$

by the definition of $\left(U_{q}\right)_{q \in \mathbb{Q}}$ outside $\mathbb{I}$.

(3) $\rightarrow$ (2): Trivial.

Definition 6.4 Let $\mathcal{S}$ be a formal topology, and let $U, V \subseteq S$. A scale $\left(U_{q}\right)_{q \in \mathbb{I}}$ from $U$ to $V$ is finitary if $U_{q} \in \operatorname{Fin}(S)$ for all $q \in \mathbb{I}$. For any $U, V \subseteq S$, the set of finitary scales from $U$ to $V$ is denoted by $\operatorname{Sc}_{\mathrm{Fin}}(U, V)$. Explicitly, $\operatorname{Sc}_{\mathrm{Fin}}(U, V)$ is the following set:

$$
\{F \in \mathbb{I} \rightarrow \operatorname{Fin}(S) \mid U \triangleleft F(0) \& F(1) \triangleleft V \&(\forall p, q \in \mathbb{I}) p<q \rightarrow F(p) \lll F(q)\} .
$$

We write $\operatorname{Sc}_{\text {Fin }}(a, b)$ for $\operatorname{Sc}_{\text {Fin }}(\{a\},\{b\})$.

The following is a special case of the interpolation property of way below relations.

Lemma 6.5 Let $\mathcal{S}$ be a compact regular formal topology. For any $U, V \subseteq S$ such that $U \lll V$, there exists $W \in \operatorname{Fin}(S)$ such that $U \lll W \lll V$.

Proof See, eg Curi [8, Lemma 3.7].

From Lemma 6.5, the following proposition is intuitively clear. It is a special case of Urysohn's lemma for locale; see Johnstone [11, Chapter IV, Proposition 1.6]. The proof requires Dependent Choice.

Proposition 6.6 Let $\mathcal{S}$ be a compact regular formal topology. Then, for any $U, V \subseteq S$ such that $U \lll V$, there exists a finitary scale from $U$ to $V$.

Proof See Curi [7, Proposition 2.4] where he gave a proof of a similar property for normal formal topologies. His proof requires Relativised Dependent Choice, but for compact regular formal topologies, Dependent Choice suffices. This is because we only need to deal with finitary scales, and $\operatorname{Sc}_{\text {Fin }}(U, V)$ is a set for each $U, V \subseteq S$. 
Proposition 6.6 motivates the following definition.

Definition 6.7 A formal topology $\mathcal{S}$ is compact enumerably completely regular if $\mathcal{S}$ is compact and there exist functions wc: $S \rightarrow \operatorname{Pow}(S)$ and sc $\in \prod_{(a, b) \in \overline{\mathrm{wc}}} \operatorname{Sc}_{\text {Fin }}(a, b)$ such that wc satisfies the two conditions of regularity in Definition 2.20, and moreover, the relation $\overline{\mathrm{wc}} \subseteq S \times S$ associated with wc is countable, ie there exists a surjection $f: \mathbb{N} \rightarrow \overline{\mathrm{wc}}$. The function sc is called a choice of scale for wc.

Remark 6.8 Curi [7] defined the notion of enumerably completely regular formal topologies, which is obtained by omitting the compactness and the finiteness condition on scales from Definition 6.7. Since our aim is to find a point-free characterisation of Bishop compact metric spaces, we have put compactness and enumerable complete regularity into one definition. See the pioneering work by Curi [7] and our subsequent paper [12] for further properties of enumerably completely regular formal topologies.

Lemma 6.9 The localic completion $\mathcal{M}(X)$ of a compact metric space $X$ is isomorphic to an overt compact enumerably completely regular formal topology.

Proof Let $X=(X, \rho)$ be a compact metric space, and let $Y \subseteq X$ be a countable dense subset of $X$. By Theorem 3.3, $\mathcal{M}(X)$ and $\mathcal{M}(Y)$ are isomorphic. Hence, without loss of generality, we may assume that $X$ is countable. Since $M_{X}=\bigcup_{\varepsilon \in \mathbb{Q}^{>0}} \mathcal{C}_{\varepsilon}$, and the set $\mathcal{C}_{\varepsilon}$ is countable for each $\varepsilon \in \mathbb{Q}^{>0}, M_{X}$ is countable. By (M1), we have

$$
\mathrm{b}(x, \varepsilon) \triangleleft_{X}\left\{\mathrm{~b}(x, \delta) \in M_{X} \mid \delta<\varepsilon\right\}
$$

for each $\mathrm{b}(x, \varepsilon) \in M_{X}$. Since $a<_{X} b$ implies $a \lll b$ for any $a, b \in M_{X}$, we define a function wc: $M_{X} \rightarrow \operatorname{Pow}\left(M_{X}\right)$ by

$$
\mathrm{wc}(\mathrm{b}(x, \varepsilon)) \stackrel{\text { def }}{=}\left\{\mathrm{b}(x, \delta) \in M_{X} \mid \delta<\varepsilon\right\} .
$$

The set $\operatorname{wc}(\mathrm{b}(x, \varepsilon))$ is countable by the standard enumeration of the rational interval $(0, \varepsilon)$. Therefore, the relation $\overline{\mathrm{wC}}$ is countable.

Moreover, for any $\mathrm{b}(x, \delta) \in \mathrm{wc}(\mathrm{b}(x, \varepsilon))$, we can choose an order preserving bijection $\varphi: \mathbb{I} \rightarrow[\delta, \varepsilon] \cap \mathbb{Q}$. Then, the family $(\{\mathrm{b}(x, \varphi(q))\})_{q \in \mathbb{I}}$ is a finitary scale from $\{\mathrm{b}(x, \delta)\}$ to $\{\mathrm{b}(x, \varepsilon)\}$. Thus, we can define a function $\mathrm{sc} \in \prod_{(a, b) \in \overline{\mathrm{wc}}} \operatorname{Sc}_{\mathrm{Fin}}(a, b)$ which assigns to each $(a, b) \in \overline{\mathrm{WC}}$ a finitary scale from $\{a\}$ to $\{b\}$ as described above.

Since $X$ is compact, $\mathcal{M}(X)$ is compact by Theorem 3.8. Therefore, $\mathcal{M}(X)$ is an overt compact enumerably completely regular formal topology with the function wc: $M_{X} \rightarrow \operatorname{Pow}\left(M_{X}\right)$ and the choice of scale sc $\in \prod_{(a, b) \in \overline{\mathrm{wc}}} \operatorname{Sc}_{\mathrm{Fin}}(a, b)$ for wc. 
Finally, we can give a point-free characterisation of Bishop compact metric spaces. Theorem 6.10 can be seen as a point-free version of Urysohn's metrisation theorem for overt compact enumerably completely regular formal topologies.

Theorem 6.10 Let $\mathcal{S}$ be a formal topology. Then, the following are equivalent.

(1) $\mathcal{S}$ is isomorphic to an overt compact enumerably completely regular formal topology.

(2) $\mathcal{S}$ is isomorphic to a compact overt subtopology of $\prod_{n \in \mathbb{N}} \mathcal{I}[0,1]$.

(3) $\mathcal{S}$ is isomorphic to the localic completion of some compact metric space.

Proof (1) $\rightarrow(2)$ : Without loss of generality, suppose that $\mathcal{S}$ is an overt compact enumerably completely regular formal topology. Then, there exist wc: $S \rightarrow \operatorname{Pow}(S)$, a choice of scale sc $\in \prod_{(a, b) \in \overline{\mathrm{wc}}} \operatorname{Sc}_{\text {Fin }}(a, b)$ for wc and a surjection $f: \mathbb{N} \rightarrow \overline{\mathrm{Wc}}$, as described in Definition 6.7. We write $\left(a_{n}, b_{n}\right)$ for $f(n)$. Then, the composition sc $\circ f$ assigns to each $n \in \mathbb{N}$, a finitary scale $(\operatorname{sc} \circ f)(n)$ from $\left\{a_{n}\right\}$ to $\left\{b_{n}\right\}$. By Proposition 6.3, each $n \in \mathbb{N}$ determines a formal topology map $r_{n}: \mathcal{S} \rightarrow \mathcal{I}[0,1]$ such that

$$
\begin{gathered}
r_{n}^{-}(0, \infty) \downarrow a_{n} \triangleleft \emptyset, \\
r_{n}^{-}(-\infty, 1) \triangleleft b_{n} .
\end{gathered}
$$

Let $r: \mathcal{S} \rightarrow \prod_{n \in \mathbb{N}} \mathcal{I}[0,1]$ be the canonical formal topology map which commutes with $r_{n}: \mathcal{S} \rightarrow \mathcal{I}[0,1]$ for each $n \in \mathbb{N}$. We show that $r$ is an embedding, ie $a \triangleleft r^{-} r^{-*} \mathcal{A}\{a\}$ for each $a \in S$. Let $a \in S$ and $b \in \operatorname{wc}(a)$. Then, there exists $n \in \mathbb{N}$ such that $f(n)=(b, a)$. Define

$$
\begin{aligned}
S_{a} & \stackrel{\text { def }}{=}\left\{k \in \mathbb{N} \mid r_{k}{ }^{-}(-\infty, 1) \triangleleft a\right\}, \\
V & \stackrel{\text { def }}{=} \bigcup_{k \in S_{a}} p_{k}{ }^{-}(-\infty, 1),
\end{aligned}
$$

where $p_{k}$ is the $k$ th projection $p_{k}: \prod_{n \in \mathbb{N}} \mathcal{I}[0,1] \rightarrow \mathcal{I}[0,1]$. Note that $r^{-} V=\mathcal{S}$ $\bigcup_{k \in S_{a}} r^{-} p_{k}{ }^{-}(-\infty, 1)=\mathcal{S} \bigcup_{k \in S_{a}} r_{k}^{-}(-\infty, 1) \triangleleft a$. Thus, $V \subseteq r^{-*} \mathcal{A}\{a\}$. Since $S \triangleleft r_{n}{ }^{-}(-\infty, 1) \cup r_{n}{ }^{-}(0, \infty)$, we have

$$
\begin{aligned}
b & \triangleleft\left(r_{n}{ }^{-}(-\infty, 1) \cup r_{n}{ }^{-}(0, \infty)\right) \downarrow b \\
& \triangleleft\left(r_{n}{ }^{-}(-\infty, 1) \downarrow b\right) \cup\left(r_{n}{ }^{-}(0, \infty) \downarrow b\right) \\
& \triangleleft r_{n}{ }^{-}(-\infty, 1) \cup \emptyset \\
& \triangleleft r^{-} p_{n}{ }^{-}(-\infty, 1) \subseteq r^{-} V .
\end{aligned}
$$

Hence, $a \triangleleft \operatorname{wc}(a) \triangleleft r^{-} r^{-*} \mathcal{A}\{a\}$. Therefore, $r$ is an embedding. Then, the conclusion is immediate. 
$(2) \rightarrow(3)$ : Suppose that $\mathcal{S}$ is isomorphic to a compact overt subtopology of $\prod_{n \in \mathbb{N}} \mathcal{I}[0,1]$. Since $\mathcal{M}([0,1]) \cong \mathcal{I}[0,1]$ by Example 4.18 and $[0,1]$ is an inhabited compact metric space, we have $\prod_{n \in \mathbb{N}} \mathcal{I}[0,1] \cong \mathcal{M}\left(\prod_{n \in \mathbb{N}}[0,1]\right)$ by Theorem 5.4. Since $\prod_{n \in \mathbb{N}}[0,1]$ is a compact metric space, $\mathcal{S}$ is isomorphic to the localic completion of some compact metric space by Corollary 4.17.

(3) $\rightarrow(1)$ : By Lemma 6.9.

Remark 6.11 It is straightforward to show that every compact overt subtopology of $\prod_{n \in \mathbb{N}} \mathcal{I}[0,1]$ is overt compact enumerably completely regular without using any choice principle. Hence, the equivalence (1) $\leftrightarrow$ (2) and the implication (3) $\rightarrow$ (1) do not depend on any choice principle.

However, the proof of the implication (2) $\rightarrow$ (3) contains essential use of Dependent Choice. Specifically, Corollary 4.17 depends on Lemma 4.2, whose proof requires Dependent Choice. This use of choice principles seems to be crucial.

\section{Acknowledgement}

The author is grateful for the anonymous referees for useful suggestions. In particular, Proposition 2.14 was suggested by one of the referees, which significantly simplifies our presentation of Section 4. He also thanks Hajime Ishihara and Takako Nemoto for useful comments on an earlier draft. The author was funded by the Japan Society for the Promotion of Science (JSPS) as a research fellow.

\section{References}

[1] P Aczel, M Rathjen, Notes on Constructive Set Theory, Technical Report 40, Institut Mittag-Leffler (2000/2001)

[2] E Bishop, Foundations of Constructive Analysis, McGraw-Hill, New York (1967)

[3] M Bunge, J Funk, Constructive theory of the lower power locale, Math. Structures Comput. Sci. 6 (1996) 69-83; doi: 10.1017/S0960129500000876

[4] J Cederquist, T Coquand, Entailment relations and distributive lattices, from: "Logic Colloquium '98', (S Buss, P Hajek, P Pudlak, editors), Lecture Notes in Logic 13, Association of Symbolic Logic (2000) 127-139

[5] T Coquand, E Palmgren, B Spitters, Metric complements of overt closed sets, MLQ Math. Log. Q. 57 (2011) 373-378; doi: 10.1002/malq.201010011 
[6] T Coquand, G Sambin, J Smith, S Valentini, Inductively generated formal topologies, Ann. Pure Appl. Logic 124 (2003) 71-106; doi: 10.1016/S0168-0072(03)00052-6

[7] G Curi, Constructive metrisability in point-free topology, Theoret. Comput. Sci. 305 (2003) 85-109; doi: 10.1016/S0304-3975(02)00699-0

[8] G Curi, On the collection of points of a formal space, Ann. Pure Appl. Logic 137 (2006) 126-146; doi: 10.1016/j.apal.2005.05.019

[9] G Curi, Exact approximations to Stone-Čech compactification, Ann. Pure Appl. Logic 146 (2007) 103-123; doi: 10.1016/j.apal.2006.12.004

[10] C Fox, Point-set and Point-free Topology in Constructive Set Theory, PhD thesis, University of Manchester (2005)

[11] P T Johnstone, Stone Spaces, Cambridge University Press (1982)

[12] T Kawai, A point-free characterisation of Bishop locally compact metric spaces, Journal of Logic \& Analysis 9 (2017) 1-41; doi: 10.4115/jla.2017.9.c2

[13] S Negri, D Soravia, The continuum as a formal space, Arch. Math. Logic 38 (1999) 423-447; doi: 10.1007/s001530050149

[14] E Palmgren, A constructive and functorial embedding of locally compact metric spaces into locales, Topology Appl. 154 (2007) 1854-1880; doi: 10.1016/j.topol.2007.01.018

[15] G Sambin, Intuitionistic formal spaces - a first communication, from: "Mathematical Logic and its Applications", (D G Skordev, editor), volume 305, Plenum Press, New York (1987) 187-204; doi: 10.1007/978-1-4613-0897-3_12

[16] B Spitters, Locatedness and overt sublocales, Ann. Pure Appl. Logic 162 (2010) 36-54; doi: 10.1016/j.apal.2010.07.002

[17] S Vickers, Localic completion of generalized metric spaces I, Theory Appl. Categ. 14 (2005) 328-356

[18] S Vickers, Some constructive roads to Tychonoff, from: "From Sets and Types to Topology and Analysis: Towards Practicable Foundations for Constructive Mathematics", (L Crosilla, P Schuster, editors), Oxford Logic Guides 48, Oxford University Press (2005) 223-238; doi: 10.1093/acprof:oso/9780198566519.003.0014

[19] S Vickers, Sublocales in formal topology, J. Symbolic Logic 72 (2007) 463-482; doi: $10.2178 /$ jsl/1185803619

School of Information Science, Japan Advanced Institute of Science and Technology

1-1 Asahidai, Nomi, Ishikawa 923-1292, Japan

tatsuji.kawai@jaist.ac.jp

Received: 1 October $2015 \quad$ Revised: 12 March 2017 